\title{
Fructose driven glycolysis supports anoxia resistance in the naked mole-rat
}

Thomas J. Park ${ }^{1 \text { ** }}$, Jane Reznick ${ }^{2, \ddagger}$, Bethany L. Peterson ${ }^{1}$, Gregory Blass ${ }^{1}$, Damir Omerbašić ${ }^{2}$, Nigel C. Bennett ${ }^{3}$, P. Henning J.L. Kuich ${ }^{4}$, Christin Zasada ${ }^{4}$, Brigitte M. Browe ${ }^{1}$, Wiebke Hamann $^{5}$, Daniel T. Applegate ${ }^{1}$, Michael H Radke ${ }^{5,10}$, Tetiana Kosten ${ }^{2}$, Heike Lutermann ${ }^{3}$, Victoria Gavaghan ${ }^{1}$, Ole Eigenbrod ${ }^{2}$, Valérie Bégay ${ }^{2}$, Vince G. Amoroso ${ }^{1}$, Vidya Govind ${ }^{1}$, Richard D. Minshall ${ }^{7}$, Ewan St. J. Smith ${ }^{8}$, John Larson ${ }^{9}$, Michael Gotthardt ${ }^{5,10}$, Stefan Kempa ${ }^{4}$, Gary R. Lewin ${ }^{2,11 *}$

${ }^{1}$ Laboratory of Integrative Neuroscience, Department of Biological Sciences, University of Illinois at Chicago, Chicago, Illinois, United States of America,

${ }^{2}$ Molecular Physiology of Somatic Sensation, Max Delbrück Center for Molecular Medicine, Berlin, Germany,

${ }^{3}$ Department of Zoology and Entomology, University of Pretoria, Pretoria, Republic of South Africa

${ }^{4}$ Integrative Proteomics and Metabolomics, Berlin Institute for Medical Systems Biology, Max Delbrück Center for Molecular Medicine, Berlin, Germany

${ }^{5}$ Neuromuscular and Cardiovascular Cell Biology, Max Delbrück Center for Molecular Medicine, Berlin, Germany

${ }^{7}$ Departments of Anesthesiology and Pharmacology, University of Illinois at Chicago, Chicago, Illinois, United States of America

${ }^{8}$ Department of Pharmacology, University of Cambridge, Cambridge CB2 1PD, United Kingdom.

${ }^{9}$ Department of Psychiatry, University of Illinois at Chicago, Chicago, Illinois, United States of America

${ }^{10}$ DZHK partner site Berlin, Germany; ${ }^{11}$ Excellence cluster Neurocure, Charité Universitätsmedizin Berlin, Germany

\$ These authors contributed equally

*Correspondence to glewin@mdc-berlin.de or tpark@uic.edu 


\begin{abstract}
The African naked mole-rats' (Heterocephalus glaber) social and subterranean lifestyle generates a hypoxic niche. Under experimental conditions naked mole-rats tolerate hours of extreme hypoxia and survive 18 minutes of total oxygen deprivation (anoxia) without apparent injury. During anoxia the naked mole-rat switches to anaerobic metabolism fueled by fructose which is actively accumulated and metabolized to lactate in the brain. Global expression of the GLUT5 fructose transporter and high levels of ketohexokinase (KHK) were identified as molecular signatures of fructose metabolism. Fructose-driven glycolytic respiration in naked mole-rat tissues avoids feedback inhibition of glycolysis via phosphofructokinase, supporting viability. The metabolic rewiring of glycolysis can circumvent the normally lethal effects of oxygen-deprivation a mechanism that could be harnessed to minimize hypoxic damage in human disease.
\end{abstract}

One Sentence Summary: Fructose can drive sufficient anaerobic glycolysis in hypoxic naked mole-rat tissues to preserve tissue physiology during oxygen deprivation 
In all kingdoms of life, extreme habitats drives adaptive change to enable species to exploit challenging environments. One challenge faced by subterranean mammals that inhabit confined spaces is an atmosphere low in oxygen $\left(\mathrm{O}_{2}\right)$ and high in carbon dioxide $\left(\mathrm{CO}_{2}\right)$. Here we studied adaptation to low $\mathrm{O}_{2} /$ high $\mathrm{CO}_{2}$ using naked mole-rats (Heterocephalus glaber) (Fig.1A), as this eusocial rodent combines a subterranean lifestyle with large colony sizes of up to 280 members (1-3). Carbon dioxide levels in naked mole-rat burrows can be orders of magnitude higher than in surface air (7-10\%) (4). Correspondingly, naked mole-rats only start to display behavioral avoidance, hyperventilation or tissue acidosis when $\mathrm{CO}_{2}$ levels reach $10 \%$ (fig. S1A-E). Even a 5 hour exposure to $80 \% \mathrm{CO}_{2}\left(20 \% \mathrm{O}_{2}\right)$ was not lethal for naked mole-rats (fig S1F). Oxygen levels are low in the burrows of subterranean mammals (as low as $6 \%)(5,6)$ and the mass huddling behavior of naked mole-rats may exacerbate their exposure to hypoxic stress.

To investigate the molecular mechanisms that allow naked mole-rats to overcome hypoxic stress we challenged them to controlled hypoxia using atmospheric chambers (Fig 1B,C), approved by local ethics committees. Remarkably, naked mole-rats tolerate a chronic hypoxic environment of $5 \% \mathrm{O}_{2}$ for 5 hours with no apparent ill effects, whereas mice (Mus musculus) died in less than 15 min (Fig. 1B). We next exposed animals to $0 \% \mathrm{O}_{2}$ in a chamber flushed with $\mathrm{N}_{2}(10 \mathrm{l} / \mathrm{min})$. Respiration in mice ceased on average $45 \pm 5 \mathrm{~s}$ after entering the chamber, and when re-exposed to normoxia $20 \mathrm{~s}$ later $(\mathrm{n}=4)$, none recovered (Fig. 1C). Like mice, naked mole-rats rapidly lost consciousness after exposure to $0 \% \mathrm{O}_{2}(\sim 30 \mathrm{~s})$, but unlike mice, continued to make sporadic breathing attempts for several minutes (mean $250 \pm 2.2 \mathrm{~s}$; $\mathrm{n}=4$ ) (Fig. 1D). After respiration ceased naked mole-rats were left in $0 \% \mathrm{O}_{2}$ for an additional minute. Surprisingly, all naked mole-rats $(\mathrm{n}=4)$ started breathing within seconds upon exposure to room air (Fig. 1C), and all rejoined their colony with no sign of neurological or behavioral deficits. In further cohorts, naked 
mole-rats recovered from fixed $10 \mathrm{~min}$ (fig. S2A,B) and $18 \mathrm{~min}$ (Fig. 1D,E) exposures, but never from a 30 min exposure (Fig. 1F). Respiratory attempts stopped after $\sim 7$ min, but resumed after 10 min (Fig. 1D). Remarkably, heart rate dropped within 2 min from a baseline of around 200 beats per minute $(\mathrm{bpm})(7)$ to a steady $50 \mathrm{bpm}$ throughout anoxia (Fig. 1E). In mice, the heart rate rapidly and continuously declined until at $\sim 6$ mins the electrocardiogram (ECG) was undetectable (Fig. 1E). In anoxic conditions circulating hemoglobin, which shows a high affinity for $\mathrm{O}_{2}$ (22), could provide a minimal $\mathrm{O}_{2}$ supply to naked mole-rat organs. Naked mole-rat body temperature was maintained constant at $30^{\circ} \mathrm{C}$ during anoxia (fig $\mathrm{S} 3 \mathrm{~B}$ ), the preferred body temperature of naked mole-rats which are poikilothermic $(8,9)$. But, warming up naked molerats to $37^{\circ} \mathrm{C}$ decreased maximum survival times to 6 mins (fig. $\mathrm{S} 3 \mathrm{C}$ ).

Experiments with isolated hearts (Langendorff preparation) exposed to hypoxia (by stopping perfusion with oxygenated buffer for 30 mins) showed that left ventricular developed pressure (LVDP) recovered almost completely to pre-ischemic values in naked-mole-rat, but not in mouse hearts (Fig 1G, Table S1). The mouse LVDP never recovered to more than $65 \%$ of baseline even when examined at $30{ }^{\circ} \mathrm{C}$. Thus, the ability of the naked mole-rat heart to continue beating under anoxia is supported by an intrinsic cardiac hypoxia resistance. Interestingly, both hypercapnia and hypoxia lead to pulmonary edema in mice, but not in naked mole-rats (fig. S4A,B)

We postulated that naked mole-rat vital organs survive oxygen deprivation with metabolic suppression similar to hibernation, torpor or suspended animation-like states (10-12). Using gas chromatography-mass spectrometry $(\mathrm{GC}-\mathrm{MS})$ based metabolomics $(13,14)$ we measured quantitative changes in metabolite concentration during anoxia (calibrations in fig. S5) and compared normoxic baseline to $40 \mathrm{~s}$ and 10 mins (mouse) or 10 and 30 mins (naked mole-rat, only) of anoxia (Fig 2A). In contrast to mice, only minor changes in the succinate/fumarate ratio 
(15) were observed in naked mole-rat tissues during anoxia, a sign of mitochondrial shut-down (fig S6). GC-MS metabolomics can resolve hexoses which allowed us to observe a specific and striking increase in fructose and sucrose, in the liver, kidney and blood of naked mole-rats 10 min into anoxia (Fig 2B,C,D; fig S7A,B). No statistically significant changes in the levels of these sugars were seen in mouse tissues during anoxia (Fig. 2D, fig S7). The unexpected appearance of high concentrations of fructose (up to $240 \mu \mathrm{M}$ in blood) and sucrose, a fructoseglucose disaccharide, (up to $1.47 \mathrm{mM}$ in blood at $30 \mathrm{mins}$, fig. S7), in anoxic tissues suggested that these sugars might fuel metabolism under hypoxic conditions. Fructose enters glycolytic metabolism after phosphorylation by ketohexokinase (KHK) and is converted to fructose-1phosphate (F1P). Fructolysis is prominent in the kidney which expresses high levels of both the more fructose selective KHK-C isoform and the less efficient KHK-A isoform (18-21). Consistently, we detected high levels of F1P in the kidney, which were unaltered after anoxia in both species (Fig. 2D). However, F1P was undetectable in normoxic brains, but appeared in significant amounts only in anoxic naked mole-rat brain indicating a switch to fructose metabolism (Fig. 2D). Surprisingly, naked mole-rats were hypoglycemic compared to mouse (mean blood glucose $3.49 \pm 0.1$ versus $6.66 \pm 0.3 \mathrm{mM}$ in mice; fig S8A (16)), but during anoxia glucose levels did not show consistent changes divergent from the mouse (fig S8). Furthermore, although there were some differences in glycogen stores between the two species these were relatively small and not consistent across all tissues (fig. S8).

Fructose can enter cells via GLUT2 and GLUT5 which belong to the SLC2A transporter family $(17,18)$. The GLUT5 (SLC2A5) protein is a highly selective fructose transporter (19) predominantly expressed in the mouse intestine and kidney, but hardly present in brain and heart (18). Using quantitative real-time PCR, we found that naked mole-rat GLUT5 mRNA (Slc2a5, 
fig. S9A) was expressed at high levels ( $>10$ fold higher than mouse) in all tissues examined including brain, heart, liver and lung (Fig. 2E). GLUT5 protein levels analyzed with Western blotting were higher in naked mole-rat heart and brain tissue compared to mouse, and levels broadly reflected mRNA levels (Fig. 2F, fig. S9C). Thus, naked mole-rat brain and cardiac tissue likely take-up fructose for glycolytic metabolism. Consistently, both KHK isoforms were dramatically upregulated in naked mole-rat heart, brain and liver tissue compared to the mouse (Fig. 2G, fig S9B).

Brain tissue from naked mole-rats shows a remarkable, intrinsic tolerance to anoxia (20). We thus tested whether naked mole-rat brains can function by utilizing fructose-fueled glycolytic metabolism. We measured field excitatory post-synaptic potentials (fEPSPs) in hippocampal slices from mouse and naked mole-rat hippocampi ( $\mathrm{n}=3$ per species) before and 60 mins after replacement of $10 \mathrm{mM}$ glucose in the buffer with $10 \mathrm{mM}$ fructose (normoxic conditions). With fructose as the sole available sugar, fEPSP amplitude declined steadily, but at different rates in mouse and naked mole-rat slices (Fig. 3A). In mouse, fEPSPs were almost undetectable 60 mins after the glucose to fructose switch, but fEPSP amplitudes in naked mole-rat slices had stabilized to $\sim 33 \%$ of control values. After slices were re-perfused with glucose containing buffer, mean fEPSP amplitudes returned to control levels in naked mole-rat slices, but only partially recovered in mouse slices (2-way ANOVA p<0.05) (Fig. 3A). We also asked if fructose could be used to fuel the isolated beating heart. Naked mole-rat or mouse hearts were perfused with KrebsHenseleit buffer containing glucose that was then switched to fructose for two periods of $60 \mathrm{~min}$. Interestingly, the LVDP of the naked mole-rat hearts remained stable during both fructose switches, whereas in the mouse heart LVDP was reduced during both fructose exposures, the 
latter was significantly different from the naked mole-rat (Fig. 3B). Thus, fructose can replace glucose as an energy source in the naked mole-rat brain and heart.

Glucose is metabolized via phosphofructokinase (PFK), a rate-limiting step subject to feedback inhibition via allosteric binding of ATP, low $\mathrm{pH}$ and downstream intermediates (21-23) (Fig. 4A). Fructose phosphorylation via KHK could bypass the PFK regulatory block, allowing continued glycolytic flux independent of cellular energy status. We used metabolic flux analysis to measure the incorporation of fructose derived carbons into glycolytic intermediates in hypoxic brain slices. Acutely isolated brain slices were incubated in media in which glucose was rapidly replaced with the stable isotope ${ }^{13} \mathrm{C}_{6}$-D-fructose $(10 \mathrm{mM})$ and kept at $32^{\circ} \mathrm{C}$ under conditions where oxygen levels were $5 \%$ (24). Metabolites were measured at $0,5,15$, and 30 mins after the switch to ${ }^{13} \mathrm{C}_{6}$-D-fructose. Similar metabolite pools were detected in both species suggesting that metabolism was at the same steady state in both species (fig. S10). We could measure significant incorporation of fructose-derived carbons in glycolytic intermediates in both species (Fig. 4AH), but the incorporation of fructose-derived carbons was both faster and larger in naked molerat compared to mouse (between 2 and 5 fold), and this was true for intermediates like dihydroxyacetone phosphate (DHAP) and glycerol-3-phosphate (Glyc-3-P) (Fig. 4B,H), but also for glycolytic end-products like pyruvate and lactate (Fig. 4D,G). There was also increased fructose-derived carbon incorporation into citrate in the naked mole-rat (Fig. 4E).

The naked mole-rat has evolved the ability to use fructose to fuel vital organs like heart and brain under near anaerobic conditions. This metabolic rewiring involves equipping metabolically active organs with transporters and enzymes that metabolize fructose to lactate using a pathway that bypasses metabolic block at PFK (Fig. 4A). Fructose and sucrose (the latter is degraded to hexose monomers) are both increased to very significant levels in naked mole-rat during anoxia. 
The source of these sugars is unknown. Fructolysis in mammals is normally largely restricted to the liver and kidney (25). A switch to fructose metabolism under hypoxic stress has been associated with cancer malignancy, metabolic syndrome, and heart failure (26-29). It is thus important to understand how naked mole-rats utilize fructose metabolism with no apparent physiological drawbacks. Molecular insights into the rewired metabolism of the naked mole-rat may help in devising novel strategies to prevent hypoxic damage associated with ischemic heart disease and stroke.

\section{References and Notes:}

1. J. U. Jarvis, Eusociality in a mammal: cooperative breeding in naked mole-rat colonies. Science (80-. ). 212, 571-573 (1981).

2. P. W. Sherman, J. U. M. Jarvis, R. D. Alexander, The Biology of the naked mole-rat (Princeton University Press, Princeton, N.J, 1991), Monographs in behavior and ecology.

3. L.-N. Schuhmacher, S. E. S. J. Husson, Zoé, The naked mole-rat as an animal model in biomedical research: current perspectives. Open Access Anim. Physiol. Volume 7, 137 (2015).

4. B. K. McNab, The Metabolism of Fossorial Rodents: A Study of Convergence. Ecology. 47, 712 (1966).

5. I. Shams, A. Avivi, E. Nevo, Oxygen and carbon dioxide fluctuations in burrows of subterranean blind mole rats indicate tolerance to hypoxic-hypercapnic stresses. Comp. Biochem. Physiol. A. Mol. Integr. Physiol. 142, 376-382 (2005).

6. H. Burda, R. Šumbera, S. Begall, in Subterranean Rodents (Springer Berlin Heidelberg, Berlin, Heidelberg, 2007; http://link.springer.com/10.1007/978-3-540-69276-8_3), pp. 21-33.

7. K. M. Grimes, A. K. Reddy, M. L. Lindsey, R. Buffenstein, And the beat goes on: maintained cardiovascular function during aging in the longest-lived rodent, the naked mole-rat. Am. J. Physiol. Heart Circ. Physiol. 307, H284-291 (2014).

8. N. C. Bennett, C. G. Faulkes, African mole-rats: ecology and eusociality (Cambridge University Press, Cambridge, 2000; http://www.amazon.de/dp/0521771994).

9. R. Buffenstein, S. Yahav, Is the naked mole-rat Hererocephalus glaber an endothermic yet poikilothermic mammal? J. Therm. Biol. 16, 227-232 (1991).

10. E. Blackstone, M. Morrison, M. B. Roth, H2S induces a suspended animation-like state in mice. Science. 308, 518 (2005).

11. J. F. Staples, J. C. L. Brown, Mitochondrial metabolism in hibernation and daily torpor: a review. J. Comp. Physiol. B. 178, 811-827 (2008). 
12. K. B. Storey, Out cold: biochemical regulation of mammalian hibernation - a mini-review. Gerontology. 56, 220-230 (2010).

13. M. Pietzke, C. Zasada, S. Mudrich, S. Kempa, Decoding the dynamics of cellular metabolism and the action of 3-bromopyruvate and 2-deoxyglucose using pulsed stable isotope-resolved metabolomics. Cancer Metab. 2, 9 (2014).

14. P. H. J. L. Kuich, N. Hoffmann, S. Kempa, Maui-VIA: a user-friendly software for visual identification, alignment, correction, and quantification of gas chromatography-mass spectrometry data. Bioinforma. Comput. Biol. 2, 84 (2015).

15. R. Narsai, M. Rocha, P. Geigenberger, J. Whelan, J. T. van Dongen, Comparative analysis between plant species of transcriptional and metabolic responses to hypoxia. New Phytol. 190, 472-487 (2011).

16. R. Buffenstein, M. Pinto, Endocrine function in naturally long-living small mammals. Mol. Cell. Endocrinol. 299, 101-11 (2009).

17. A. R. Manolescu, K. Witkowska, A. Kinnaird, T. Cessford, C. Cheeseman, Facilitated hexose transporters: new perspectives on form and function. Physiology (Bethesda). 22, 234-40 (2007).

18. V. Douard, R. P. Ferraris, Regulation of the fructose transporter GLUT5 in health and disease. Am. J. Physiol. Endocrinol. Metab. 295, E227-37 (2008).

19. C. F. Burant, J. Takeda, E. Brot-Laroche, G. I. Bell, N. O. Davidson, Fructose transporter in human spermatozoa and small intestine is GLUT5. J. Biol. Chem. 267, 14523-6 (1992).

20. J. Larson, T. J. T. Park, Extreme hypoxia tolerance of naked mole-rat brain. Neuroreport. 20, 1634-1637 (2009).

21. P. B. GARLAND, P. J. RANDLE, E. A. NEWSHOLME, CITRATE AS AN INTERMEDIARY IN THE INHIBITION OF PHOSPHOFRUCTOKINASE IN RAT HEART MUSCLE BY FATTY ACIDS, KETONE BODIES, PYRUVATE, DIABETES, AND STARVATION. Nature. 200, 169-70 (1963).

22. S. Lenzen, A fresh view of glycolysis and glucokinase regulation: history and current status. J. Biol. Chem. 289, 12189-94 (2014).

23. G. P. Dobson, E. Yamamoto, P. W. Hochachka, Phosphofructokinase control in muscle: nature and reversal of $\mathrm{pH}$-dependent ATP inhibition. Am. J. Physiol. - Regul. Integr. Comp. Physiol. 250 (1986).

24. G. Mauleon, J. F. Lo, B. L. Peterson, C. P. Fall, D. T. Eddington, Enhanced loading of Fura-2/AM calcium indicator dye in adult rodent brain slices via a microfluidic oxygenator. J. Neurosci. Methods. 216, 110-7 (2013).

25. J. Hallfrisch, Metabolic effects of dietary fructose. FASEB J. Off. Publ. Fed. Am. Soc. Exp. Biol. 4, 2652-2660 (1990).

26. A. M. Port, M. R. Ruth, N. W. Istfan, Fructose consumption and cancer: is there a connection? Curr. Opin. Endocrinol. Diabetes. Obes. 19, 367-74 (2012).

27. H. Liu et al., Fructose induces transketolase flux to promote pancreatic cancer growth. Cancer Res. 70, 6368-76 (2010). 
28. L. Tappy, K.-A. Lê, Metabolic effects of fructose and the worldwide increase in obesity. Physiol. Rev. 90, 23-46 (2010).

29. P. Mirtschink et al., HIF-driven SF3B1 induces KHK-C to enforce fructolysis and heart disease. Nature. 522, 444-9 (2015).

29. Acknowledgments: Work was supported a European Research Council (294678), the Deutsche Forschungsgemeinschaft SFB 665 and Go865/9-1, NSF (grant \#0744979), NIH (grants HL71626 and HL60678). Technical support provided by Maria Braunschweig, Franziska Kressin, Mathias Pippow and David J. Visintine. All relevant data are stored at the MDC and are available from the authors on request or are included in the manuscript and supplementary materials. The project was conceived and coordinated by GRL, TJP, JR, MG, SK, and NCB. Experiments performed by JR, BLP, GB, DO, WH, MHR, VB, RDM, BMB, JL, DTA, EStJS, VG, VGovind, VGA, NCB, TJP and GRL, RDM and EStJS. Data analysis and bioinformatics JR, HJLK, CZ, OE, TK, and SK. WH, MHR, and MG. Please contact SK for correspondence on metabolomics. The paper was written by GRL, TJP and JR with input from all authors.

\section{Figure Legends}

Figure 1 Extreme hypoxia and anoxia resistance in naked mole-rats (A) Mouse and naked mole-rat. (B, C) Time breathing in 5\% $\mathrm{O}_{2}$ (cut-off time 300 mins), or in anoxia $\left(0 \% \mathrm{O}_{2}\right.$, cut-off last breath): naked mole-rat always survived, mice did not $(\mathrm{N}=4-6$ per group $\mathrm{p}<0.01$; Fisher's exact test); time breathing in $0 \% \mathrm{O}_{2}$ was significantly different between species $\mathrm{p}<0.001$; Student's t-test. (D) Respiration and (E) heart rate during 18 min of $0 \% \mathrm{O}_{2},(\mathrm{~N}=4$ per species). (F) Survival plotted against duration of complete anoxia for mice and naked mole-rats $(\mathrm{N}=3-12$ per species and time point). (G) Left ventricular developed pressure (LVDP) measured in isolated hearts following a 30 minute period of no flow induced hypoxia and compared to 
baseline values (A-C Student's T-Test ; I. **p $<0.01 ; 2$-way ANOVA with Bonferroni post-hoc test $\mathrm{n}=3$ per group mean \pm s.e.m).

Figure 2 Fructose and sucrose in anoxia exposed naked mole-rats (A) Experimental design (B,C) Metabolic intermediates were quantified using GC-MS. (C) Increased fructose in anoxia exposed naked mole-rat kidney (chromatograms of species triplicates) (D) Quantification of fructose, sucrose and fructose-1-phosphate (F-1-P) levels (concentrations or peak intensity) before and after anoxia. $\mathrm{ND}=$ not detected. $\left(\mathrm{n}=3\right.$; error bars are s.e.m.; ${ }^{*} \mathrm{p}<0.05 ;{ }^{*} \mathrm{p}<0.01$, ${ }^{* * *} \mathrm{p}<0.001$ using a 2-WAY ANOVA with Tukey's post-hoc test) $(\mathbf{E})$ Expression level of GLUT5 mRNA transcripts in tissue in mouse and naked mole-rats using qPCR $(n=3$; error bars are s.e.m.; ${ }^{*} \mathrm{P}<0.05 ;{ }^{* * *} \mathrm{P}<0.001$; two-tailed unpaired t-test). (F) Western blot for GLUT5 in brain and heart tissues from both species (three biological replicates) (G) Bar graphs showing results of qPCR designed to detect KHK-C and KHK-A isoforms ( $\mathrm{n}=3$; error bars are s.e.m.; *P $<0.05 ; * * \mathrm{P}<0.01 ;$ two-tailed unpaired t-test).

Figure 3 Role of fructose in maintaining brain and heart function in naked mole-rats (A) Field excitatory post-synaptic potentials (fEPSP) were recorded in hippocampal brain slices. fEPSP amplitude declined to zero when fructose replaced glucose in mouse slices, but were maintained in naked mole-rat slices. Example traces shown on the left (scale bar $1 \mathrm{mV}$ and 10 msec). A two-way repeated measures ANOVA with Bonferroni post-hoc test $\left({ }^{* *} \mathrm{p}<0.01\right)$ revealed significant effects for species $[F(1,4)=19.8, p=0.0114]$ and time $[F(99,396)=43.43, p$ $<0.0001]$. The interaction between group and time was also significant $[F(99,396)=6.16, p<$ 0.0001], $\mathrm{n}=3$. (B) Left ventricular developed pressure (LVDP) was measured for isolated hearts following glucose replacement with fructose. Note that naked mole-rat LVDP was maintained 
but mouse LVDP declined, especially after a second exposure to fructose, statistical significance was calculated with a two-way ANOVA.

Figure 4 Metabolic flux of fructose metabolites in the hypoxic brain. (A) Glycolysis pathway, glucose enters brain via GLUT1 and is converted via Phosphofructokinase PFK. Fructose, enters cells via GLUT5 and is phosphorylated by KHK to fructose-1-phosphate (F1P) at a much higher efficiency than by hexokinase (HK). F1P is directly metabolized into trioses via ALDOB or ALDOC bypassing feedback inhibition. Incorporation of $13 \mathrm{C}$-fructose derived carbons was measured during acute hypoxia ( $\sim 5 \%$ oxygen) at $0,5,15$ and 30 minutes. Labelled quantities of the different metabolic intermediates (in blue) are shown; (B), Dihydroxyacetone phosphate (DHAP) (C) Phosphoglyceric acid (3PGA) (D), Pyruvate (E), Citrate (F), Succinate (G), Lactate (H), Glycerol-3-phosphate (Glyc-3-P). Glyceraldehyde-3-phosphate is abbreviated as GA3P. $\left(\mathrm{n}=3\right.$; error bars are s.e.m.; $* \mathrm{P}<0.05 ;{ }^{* * \mathrm{P}}<0.01,{ }^{* * * \mathrm{P}}<0.001$ using a 2-WAY ANOVA with a Tukey's post-hoc test). 
A

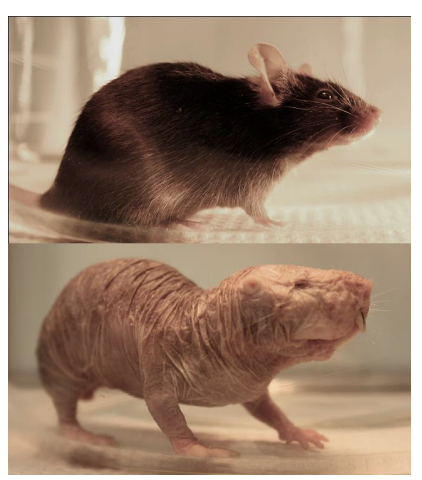

$\square$ mouse
$\square$ naked mole-rat

D

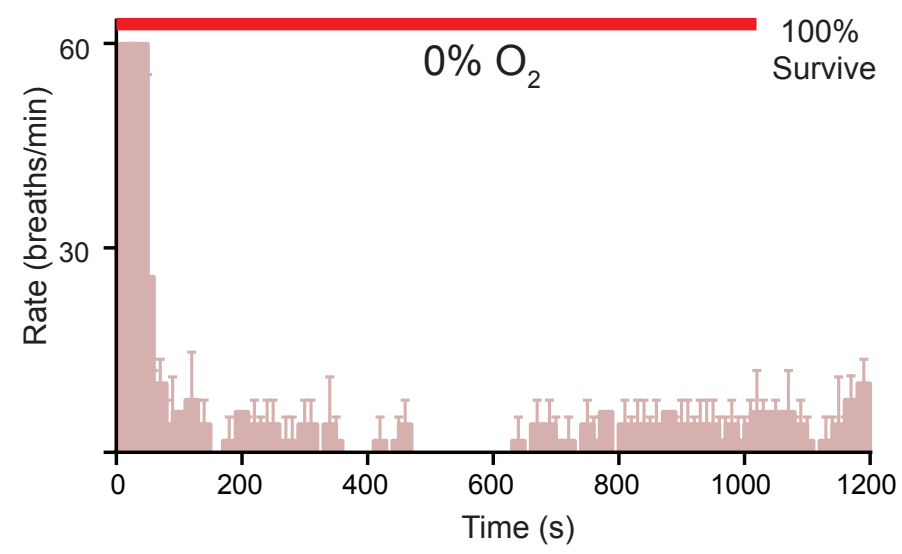

F
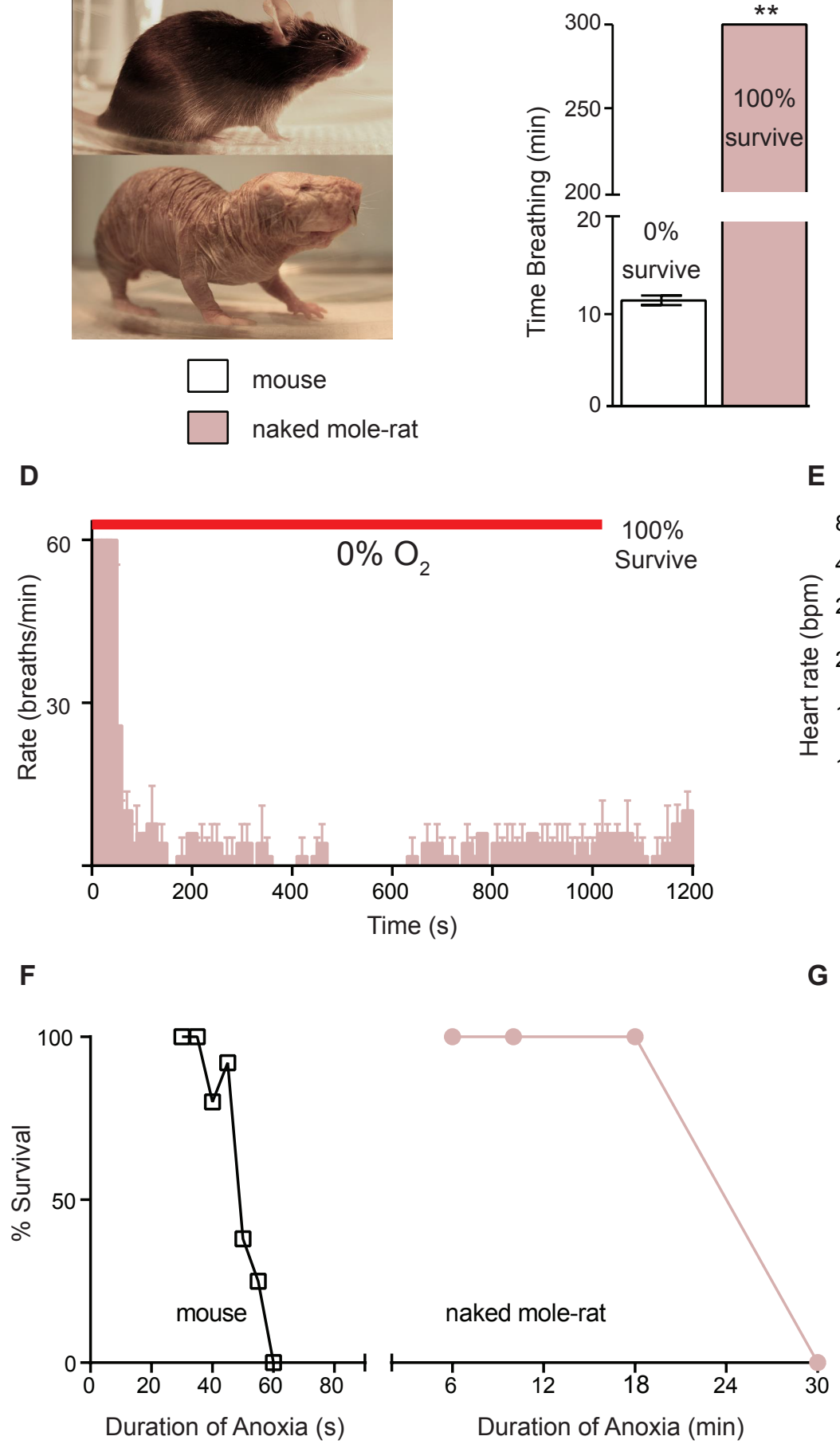

E

G
C Anoxia $\left(0 \% \mathrm{O}_{2}\right)$
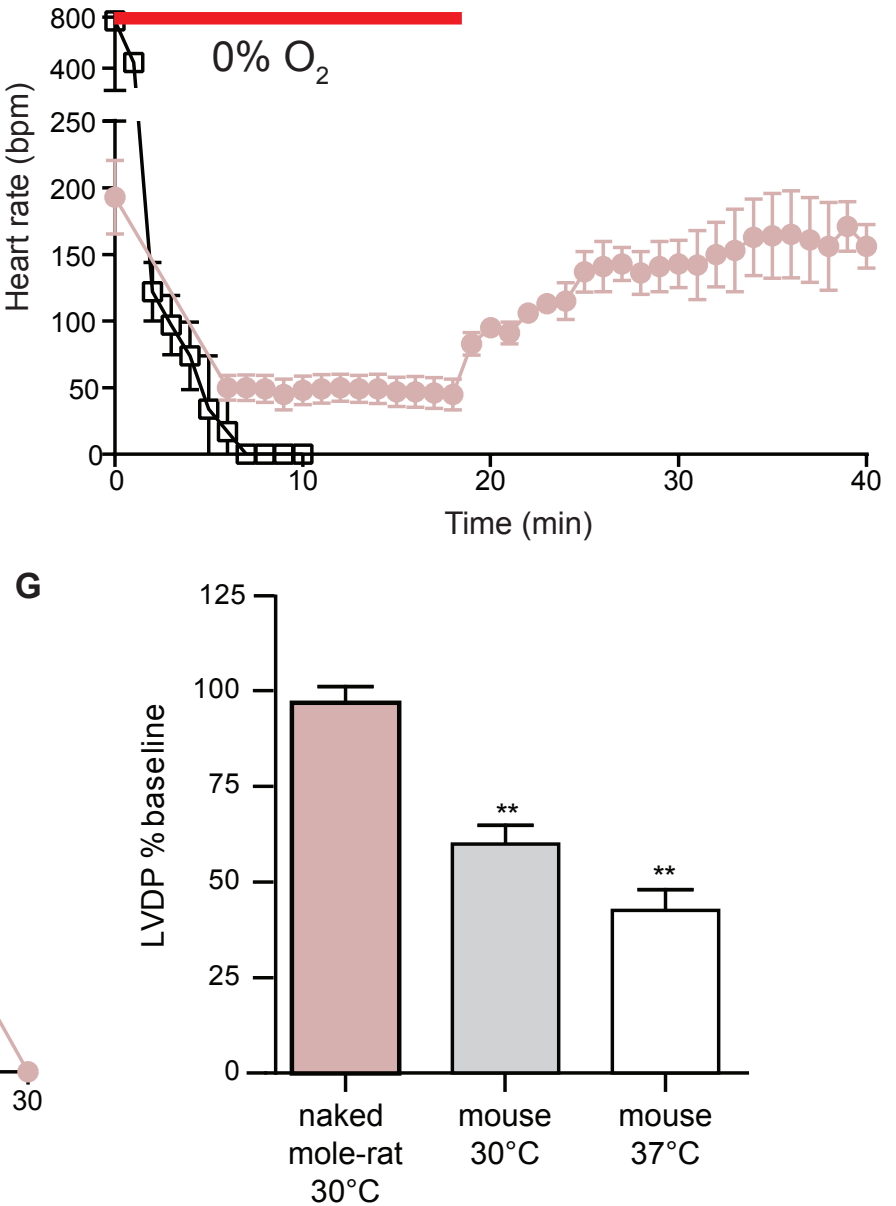
A

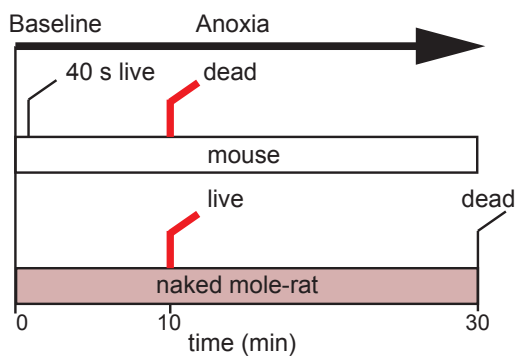

D
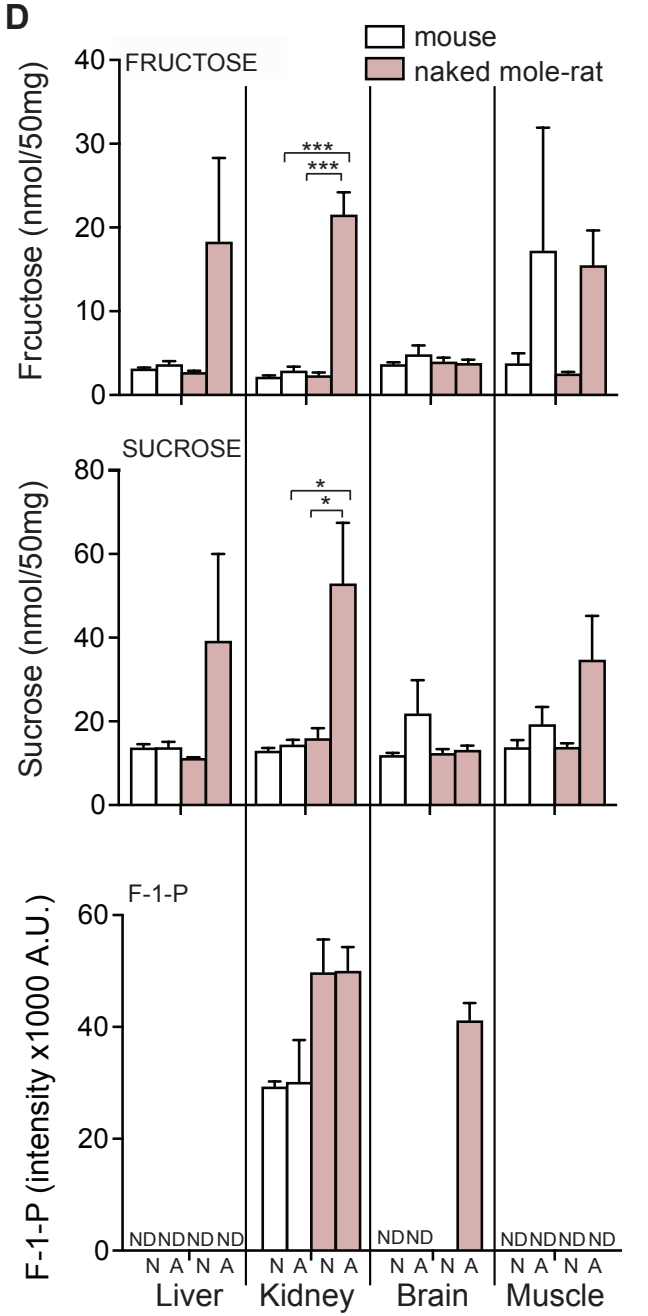

$\mathrm{N}=$ normoxia $\quad \mathrm{A}=$ anoxia
B

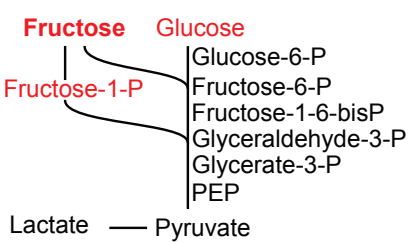

C

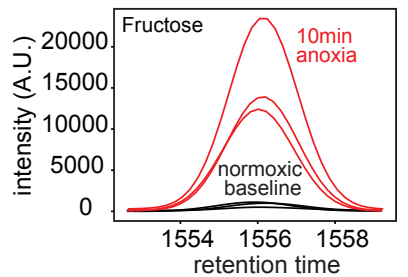

E
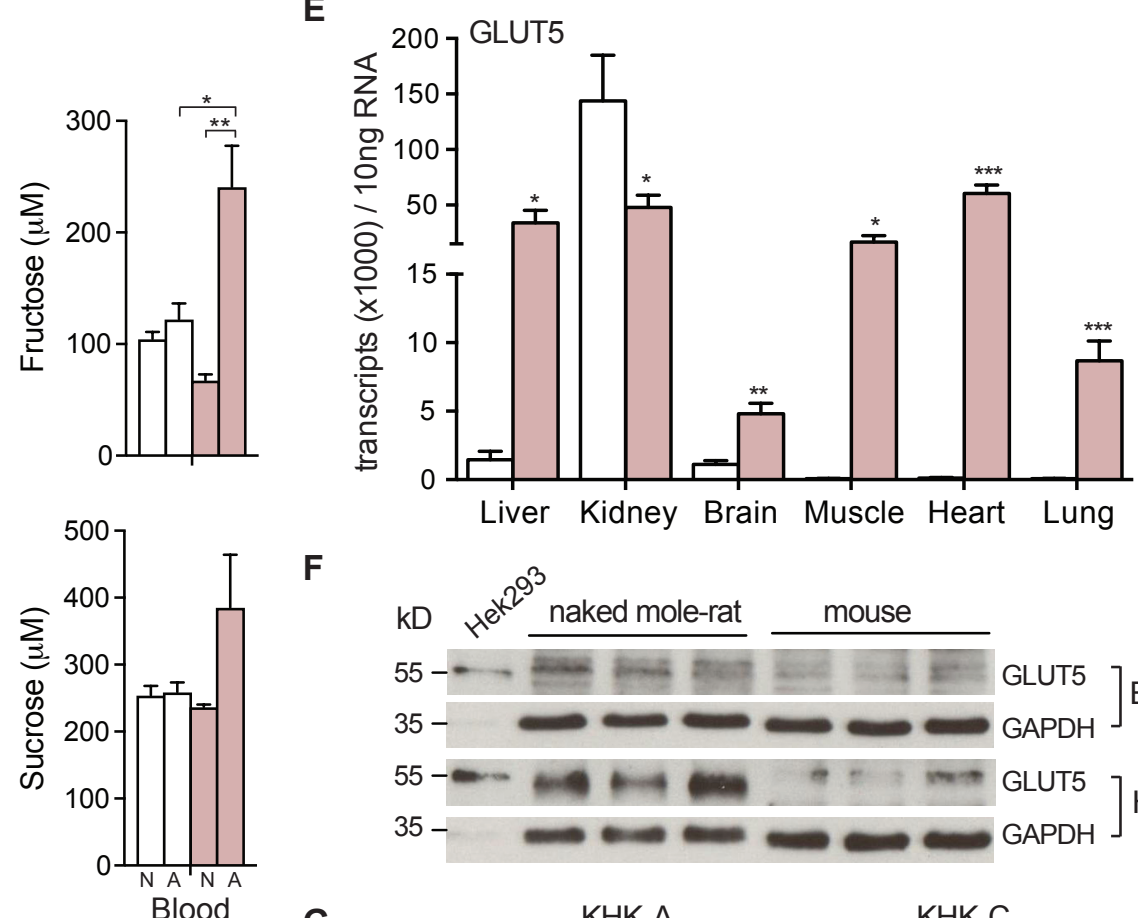

F

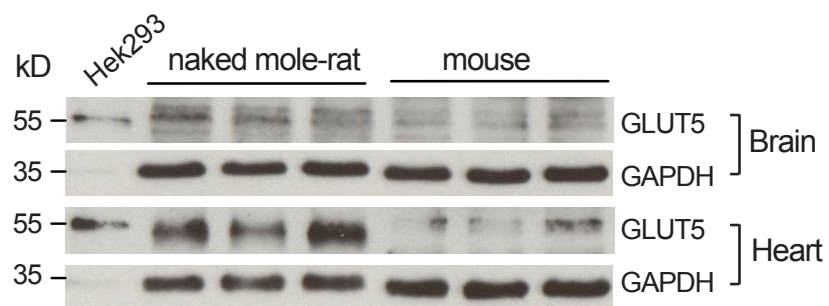

G

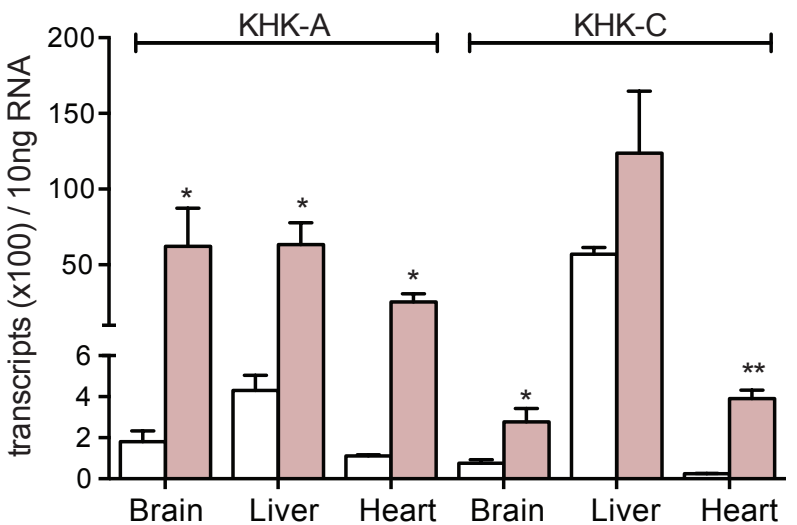


A

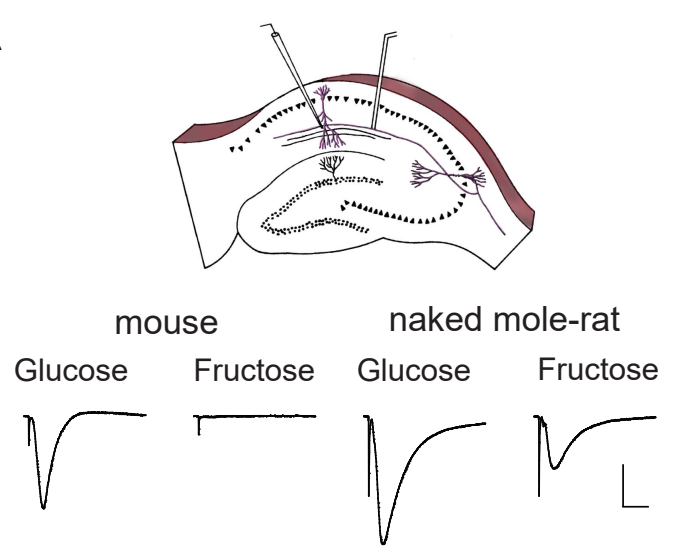

B

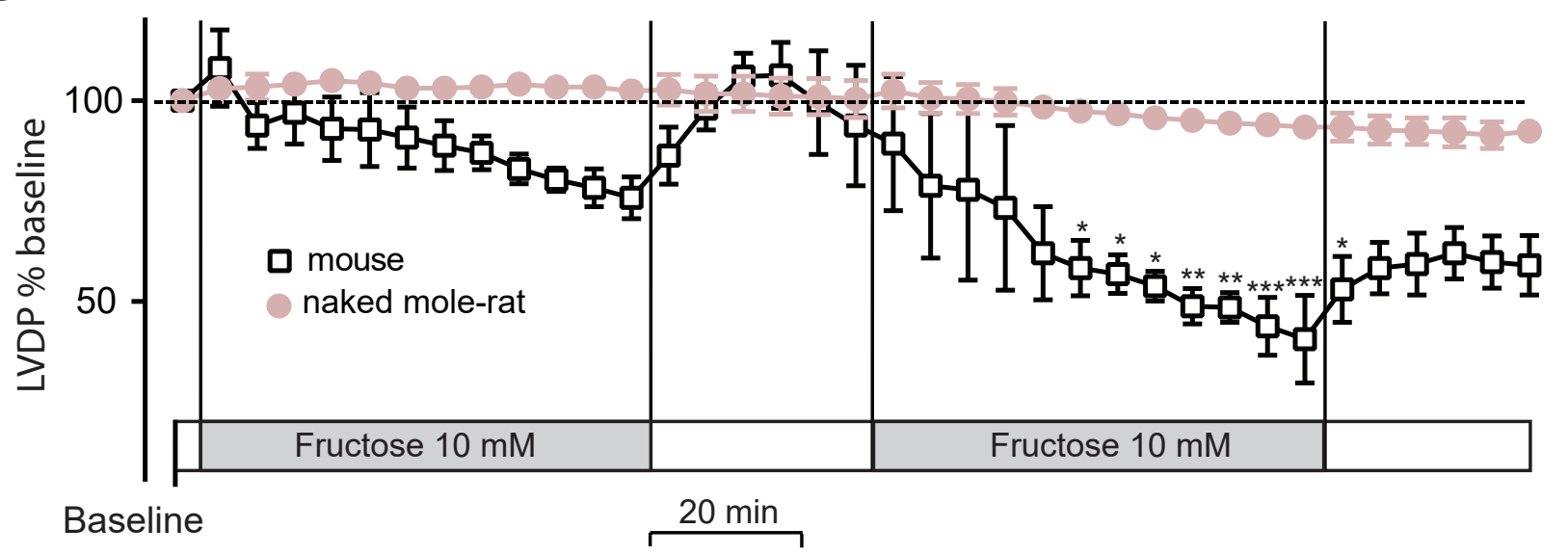

Figure 3 
A

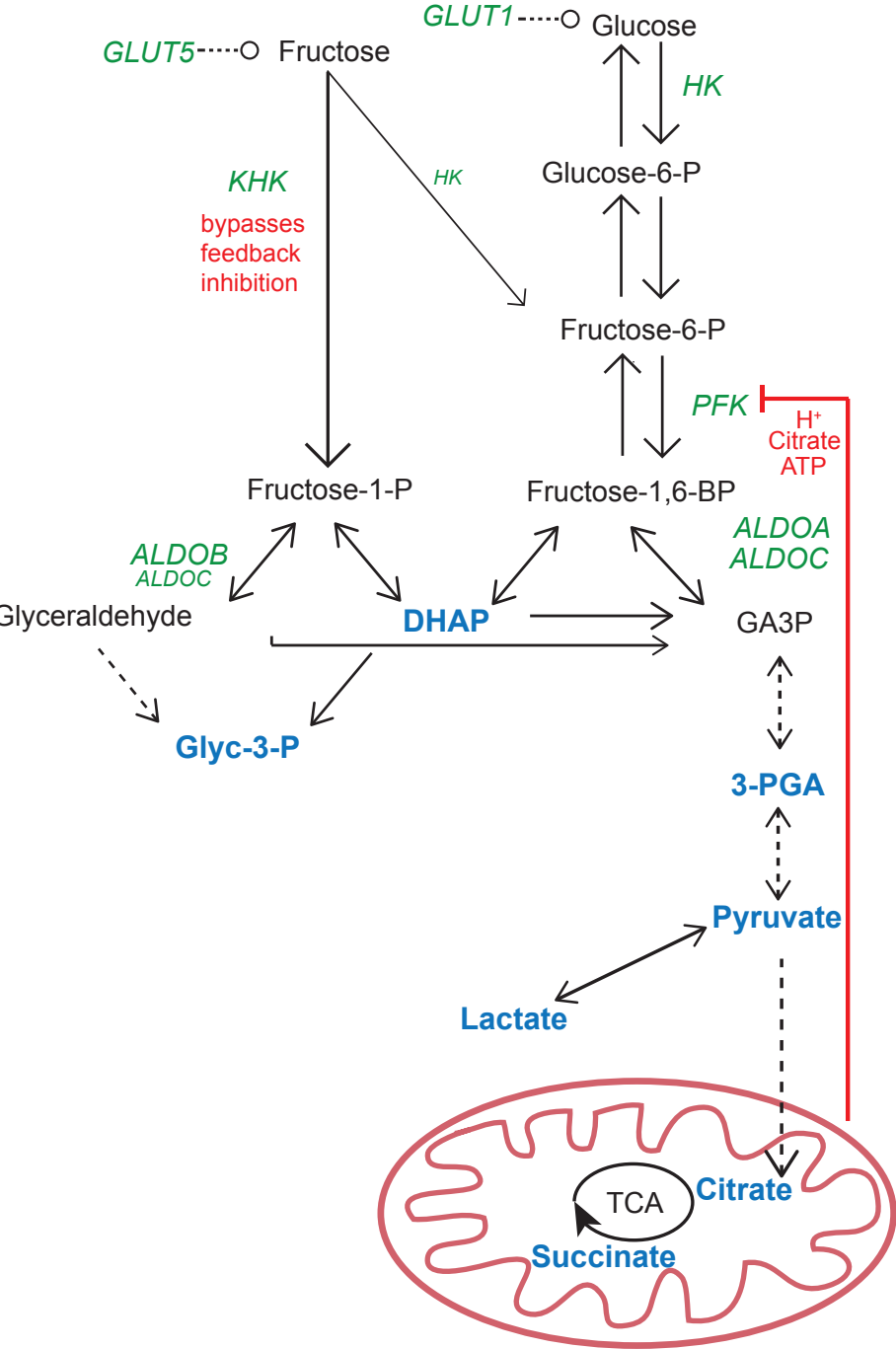

- naked mole-rat

-o- mouse

E

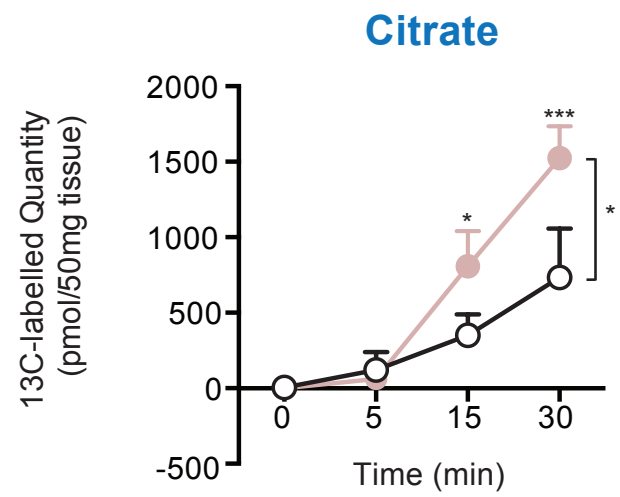

G

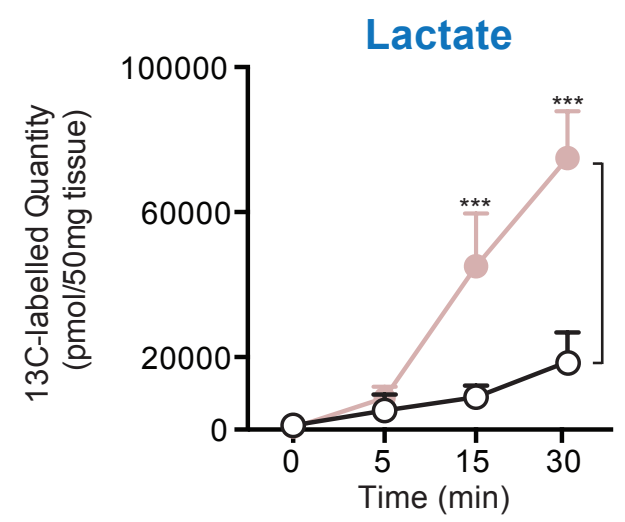

B

DHAP

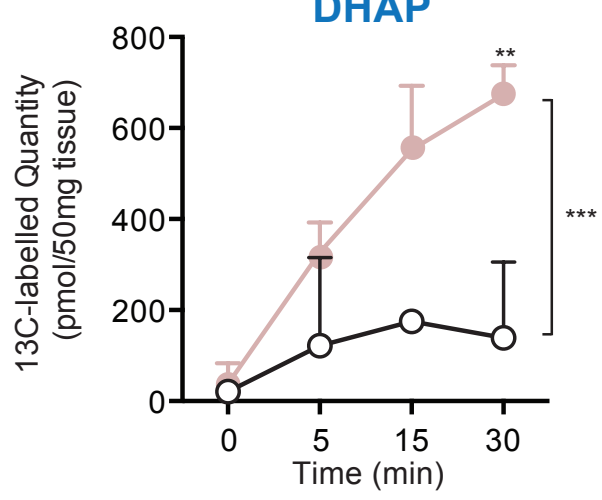

C

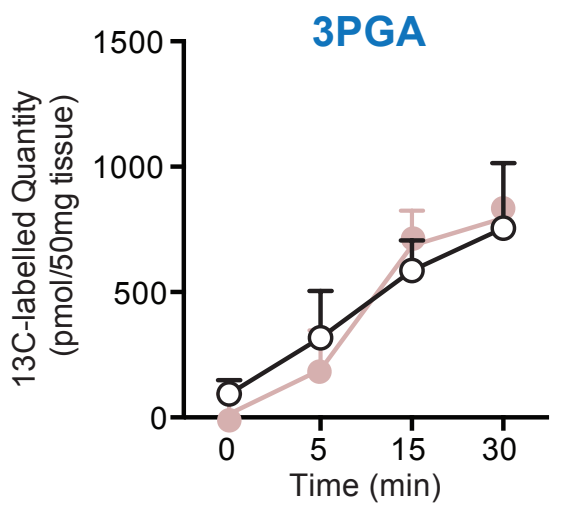

D

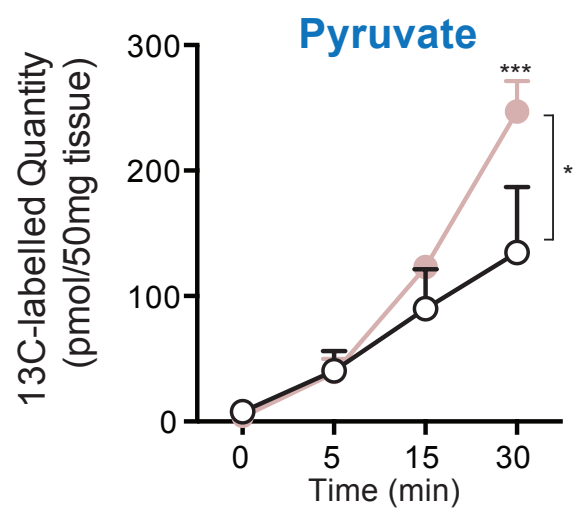

$\mathbf{F}$

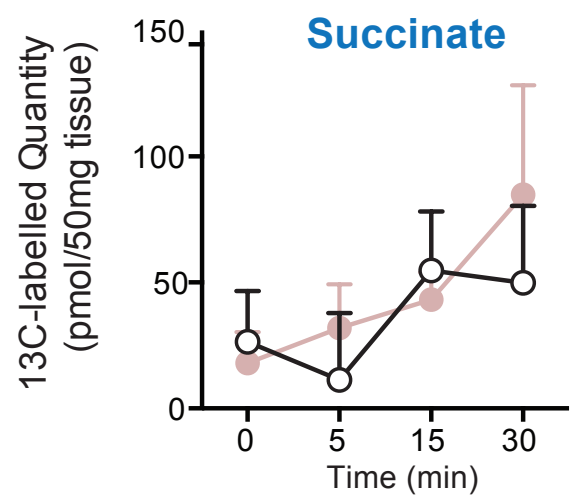

H

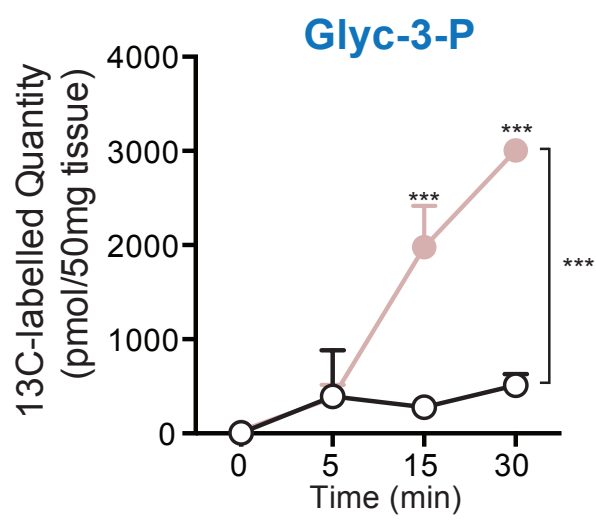




\section{Science \\ DIAAAS}

\section{Supplementary Materials for}

\section{Fructose driven glycolysis supports anoxia resistance in the naked mole- rat}

Thomas J. Park ${ }^{1 * *}$, Jane Reznick ${ }^{2, *}$, Bethany L. Peterson ${ }^{1}$, Gregory Blass ${ }^{1}$, Damir Omerbašić $^{2}$, Nigel C. Bennett ${ }^{3}$, P. Henning J.L. Kuich ${ }^{4}$, Christin Zasada ${ }^{4}$, Brigitte M. Browe $^{1}$, Wiebke Hamann ${ }^{5}$, Daniel T. Applegate ${ }^{1}$, Michael H Radke ${ }^{5,10}$, Tetiana Kosten ${ }^{2}$, Heike Lutermann ${ }^{3}$, Victoria Gavaghan ${ }^{1}$, Ole Eigenbrod ${ }^{2}$, Valerie Bégay ${ }^{2}$, Vince G. Amoroso $^{1}$, Vidya Govind ${ }^{1}$, Richard D. Minshall ${ }^{7}$, Ewan St. J. Smith ${ }^{8}$, John Larson ${ }^{9}$, Michael Gotthardt ${ }^{5,10}$, Stefan Kempa ${ }^{4}$, Gary R. Lewin ${ }^{2,11}$ *

\$ These authors contributed equally

*Correspondence to glewin@mdc-berlin.de or tpark@uic.edu

\section{This PDF file includes:}

Materials and Methods

figs. S1 to S10

Table S1

Captions for database table S1 


\section{Materials and Methods}

\section{In vivo experiments}

All animal protocols were approved by the University of Illinois at Chicago Institutional Animal Care and Use Committee, the German federal authorities (State of Berlin), or the animal use and care committee of the University of Pretoria, Republic of South Africa.

Animals were placed into a clear plastic chamber pre-filled with the desired gas mixture. Thereafter the chamber was infused continuously at 10 liters per minute. Using an Ocean Optics Foxy-PI200 probe, and an Ocean Optics sensor connected to a computer, we measured the fill time, which was, on average, $59.7 \pm 2.3$ seconds (standard error). Based on the data, we pre-filled the chamber for 120 seconds prior to introducing the animal. Each animal was video recorded for the collection of accurate timing data.

ECGs were recorded non-invasively using the ECGenie recording enclosure (Mouse Specifics, Inc., Boston, MA, USA)(1). In brief, the ECGenie system comprises a platform with embedded paw-sized AgCl ECG electrodes connected to an amplifier (e-MOUSE). Signals were collected and analysed using PowerLab and LabChart (ADInstruments, version 7). A peak detection algorithm on LabChart enabled R-wave identification using Fourier analysis and linear time-invariant digital filtering of frequencies below $3 \mathrm{~Hz}$ and above $100 \mathrm{~Hz}$ to minimize environmental signal disturbances. Only data from continuous recordings were used in the analyses. The signals were digitized at a sampling rate of

$2 \mathrm{kHz}$. Baseline heart data were acquired on conscious animals after which animals were placed in an $\mathrm{N}_{2}$ filled chamber with a continuous stream of $\mathrm{N}_{2}$ gas. Shortly after $\mathrm{N}_{2}$ exposure the animal became unconscious and ECG was recorded by placing each of the 
front paws of the unconscious animal on a single electrode. Breaths were recorded visually and counted by an observer with a manual counter.

Naked mole-rats were kept on a heated mat to maintain their body temperature at $30^{\circ}$ or $37^{\circ}$. Body temperature was measured with a digital rectal probe (Fine Science Tools TR$100)$.

Pulmonary edema measurements were made as follows. Animals were exposed to a desired gas mixture for 15 minutes. Following exposure, the animal was immediately decapitated and the lungs removed and weighed. The lungs were then dried overnight and weighed again to generate a wet-to-dry weight ratio which was used as an assay of lung edema.

\section{Isolated heart}

Perfusion and ischemia induction in isolated heart preparations: 12 week old mice and 3 to 11 year old naked mole rats were sacrificed by cervical dislocation or decapitation, respectively. Hearts were quickly removed and immersed in ice-cold modified KrebsHenseleit (KH) solution, containing: $111.8 \mathrm{mM} \mathrm{NaCl} ; 24.7 \mathrm{mM} \mathrm{NaHCO}$; $4.7 \mathrm{mM} \mathrm{KCl}$; $2.1 \mathrm{mM} \mathrm{MgSO} 4 ; 1.2 \mathrm{mM} \mathrm{KH2PO} ; 2.0 \mathrm{mM} \mathrm{CaCl2} ; 0.06$ mM EDTA; $11.1 \mathrm{mM}$ glucose. The aorta was immediately cannulated for retrograde perfusion with $\mathrm{KH}$ solution at a constant pressure of $80 \mathrm{mmHg}$. The buffer solution was saturated with 95\% O2 / $5 \%$ CO2. To measure left ventricular developed pressure (LVDP), a balloon was placed into the left ventricle through the left articular appendage and inflated to maintain a constant end diastolic pressure of $5-10 \mathrm{mmHg}$. Measurements were performed at $30{ }^{\circ} \mathrm{C}$ or $37{ }^{\circ} \mathrm{C}$ as indicated. After an adjustment time of 30 min cardiac activity was stable (baseline) 
and a global ischemia was induced by stopping the coronary flow for $30 \mathrm{~min}$. The flow was set back to normal during the re-perfusion phase for $30 \mathrm{~min}$. Recovery of cardiac function was calculated as left ventricular developed pressure (LVDP) post ischemia compared to baseline values. For fructose treatment, a second $\mathrm{KH}$ solution was substituted with $11.1 \mathrm{mM}$ fructose instead of glucose and treated as described above with a 30 minute adjustment phase before switching to fructose buffer for $60 \mathrm{~min}$. The flow was set back to glucose for $30 \mathrm{~min}$ and the last two steps were repeated. Heart rate, systolic pressure, and diastolic pressure were recorded throughout the entire measurement.

\section{Metabolites}

At each time point, animal tissues were removed and snap-frozen in liquid nitrogen. All tissues were powdered while frozen using a BioPulverizer (BioSpec Products, Cat. No. 59012N). Powdered samples were resolved in ice-cold MCW (methanol:chloroform:water, 5:2:1), vortexed, and shaken for 45 minutes at $4^{\circ} \mathrm{C}$. MilliQ water was then added ( $1 / 2$ of initial MCW volume) to induce phase separation, vortexed, and shaken for 15 minutes at $4^{\circ} \mathrm{C}$. Samples were centrifuged in a table top centrifuge at 20,000 g for 10 minutes. The resulting polar and apolar phases were collected and subsequently dried in a rotational vacuum concentrator (Martin Christ, Speed Vac RVC 2-33 CD, Cooling Trap alpha 2-4 LD plus) and then frozen at $-20{ }^{\circ} \mathrm{C}$ until derivatization.

\section{Brain Slice labelling/harvest}

Mice and naked mole-rats were decapitated and the brains were rapidly removed into icecold artificial cerebrospinal fluid (ACSF) containing (in $\mathrm{mM}$ ): $\mathrm{NaCl}, 124 ; \mathrm{KCl}, 3$; KH2PO4, 1.2; NaHCO3, 26; MgSO4, 2.5; CaCl2, 3.4; Na-ascorbate, 2; and D-glucose, 
10, gassed with $95 \% \mathrm{O} 2$ and $5 \% \mathrm{CO} 2$. The tissue was then sliced at $400 \mathrm{~mm}$ with a vibrotome. Slices were briefly washed in ACSF buffer containing no glucose. For labeling experiments $10 \mathrm{mM}$ glucose in the ACSF buffer was replaced with $10 \mathrm{mM}{ }^{13} \mathrm{C} 6$ Fructose (Sigma-Aldrich). Slices were incubated for 0, 5, 15 and 30 mins at 32 degrees after which the brains were washed 2 times in label-free buffer $(140 \mathrm{mM} \mathrm{NaCl}, 5 \mathrm{mM}$ HEPES, pH7.4) and snap frozen. Frozen brain slices were powdered and 50mg of tissue was used for extraction. The experiments were performed on three animals per species.

\section{Metabolomics sample preparation}

Metabolite extraction, derivatization, GC-MS analysis and measurement was performed as described with slight modifications (2). One milliliter ice-cold Methanol-chloroformwater MCW (5:2:1) containing $2 \mu \mathrm{g} / \mathrm{ml}$ cinnamic acid was added to $50 \mathrm{mg}$ of powdered tissue and shaken for $30 \mathrm{~min}$ at $4^{\circ} \mathrm{C}$ to separate lipid and polar intermediates, after which $500 \mathrm{uL}$ of water was added to each sample, shaken for further $10 \mathrm{~min}$ and centrifuged at maximum speed for 10 min for phase separation. Polar phase was collected and dried under a vacuum.

Derivatization was carried out by dissolving dry tissue extracts in $20 \mu$ l of methoxyamine hydrochloride solution (Sigma, $40 \mathrm{mg} / \mathrm{ml}$ in pyridine (Roth)) and incubated for $90 \mathrm{~min}$ at $30^{\circ} \mathrm{C}$ with constant shaking followed by the addition of $80 \mu \mathrm{l}$ of N-methyl-N[trimethylsilyl]trifluoroacetamide (MSTFA; Machery-Nagel, Dueren, Germany) and incubation at $37^{\circ} \mathrm{C}$ for $45 \mathrm{~min}$. The extracts were centrifuged for $10 \mathrm{~min}$ at $10,000 \times \mathrm{g}$, and aliquots of $30 \mu \mathrm{l}$ were transferred into glass vials (Th. Geyer, Berlin, Germany) for gas chromatography-mass spectrometry (GC-MS) measurement. 


\section{Retention index standards and quantification standards}

Nine alkanes (n-decane, n-dodecane, n-pentadecane, n-octadecane, n-nonadecane, ndocosane, n-octacosane, n-dotriacontane, and n-hexatriacontane) were dissolved in hexane, combined at a final concentration of $2 \mathrm{mg} / \mathrm{ml}$ and stored at $4^{\circ} \mathrm{C}$. Retention index standard was added to the solvent (MSTFA) at a final concentration of $2 \%(\mathrm{v} / \mathrm{v})$ during derivatization.

The quantification mixture was composed of 63 compounds (stock concentration 1 $\mathrm{mg} / \mathrm{ml}, 20 \% \mathrm{MeOH})$. A dilution series from 1:1, 1:2, 1:5, 1:10, 1:20, 1:50, 1:100, and 1:200 was prepared(2), portioned, dried under vacuum, and stored at $-20^{\circ} \mathrm{C}$. One set of quantification standards was prepared for GC-MS measurements and measured in technical replicates within the experiment (standards were available for $63 / 80$ metabolites measured, including all key metabolites described in the results).

\section{GC-MS measurement}

Metabolite analysis was performed on a gas chromatography coupled to time of flight mass spectrometer (Pegasus III- TOF-MS-System, LECO Corp., St. Joseph, MI, USA), complemented with an auto-sampler (MultiPurpose Sampler 2 XL, Gerstel, Mülheim an der Ruhr, Germany). The samples and quantification standards were injected in splitless and split mode (split 1:5, injection volume $1 \mu \mathrm{l}$ ) in a temperature-controlled injector (CAS4, Gerstel) with a baffled glass liner (Gerstel). The following temperature program was applied during sample injection: initial temperature of $80^{\circ} \mathrm{C}$ for $30 \mathrm{~s}$ followed by a ramp with $12^{\circ} \mathrm{C} / \mathrm{min}$ to $120^{\circ} \mathrm{C}$ and a second ramp with $7^{\circ} \mathrm{C} / \mathrm{min}$ to $300^{\circ} \mathrm{C}$ and final hold for $2 \mathrm{~min}$. Gas chromatographic separation was performed on an Agilent $6890 \mathrm{~N}$ (Agilent, Santa Clara, CA, USA), equipped with a VF-5 ms column of 30-m length, 250- 
$\mu \mathrm{m}$ inner diameter, and $0.25-\mu \mathrm{m}$ film thickness (Varian, Palo Alto, CA, USA). Helium was used as carrier gas with a flow rate of $1.2 \mathrm{ml} / \mathrm{min}$. Gas chromatography was performed with the following temperature gradient: 2-min heating at $70^{\circ} \mathrm{C}$, first temperature gradient with $5^{\circ} \mathrm{C} / \mathrm{min}$ up to $120^{\circ} \mathrm{C}$ and hold for $30 \mathrm{~s}$; subsequently, a second temperature increase of $7^{\circ} \mathrm{C} / \mathrm{min}$ up to $200^{\circ} \mathrm{C}$ and a third elevation of $12^{\circ} \mathrm{C} / \mathrm{min}$ up to $320^{\circ} \mathrm{C}$ with a hold time of $2 \mathrm{~min}$. The spectra were recorded in a mass range of 60 to 600 $\mathrm{U}$ with $20 \mathrm{spectra} / \mathrm{s}$ at a detector voltage of $1750 \mathrm{~V}$.

\section{Data analysis}

Data analysis was performed using ChromaTOF Version 4.42 (LECO) and Maui-VIA software as described previously $(2,3)$. Calibration curves were determined for each intermediate by linear regression analysis of known quantities and the detected total peak areas of the top 5 most intense ions $\mathrm{m} / \mathrm{z}$ from the quantification standards. Ions dependent on carbon-13 introduced intensity shift were complemented with their corresponding matches for the absolute quantification. Metabolite pool sizes have been calculated by assuming metabolic state and summarizing all quantities of the time-course pSIRM (pulsed stable isotope metabolomics) experiment. Only peak areas within the linear range of the calibration curve have been taken into account for the absolute quantification.

In the initial GS-MS experiment higher concentrations of glucose e.g. like those found in blood, were not included in the calibration and measured intensities fell outside of the calibration curve. To allow a robust quantification of glucose we ran higher concentration standards in a second experiment to obtain an extended calibration curve for the quantification of glucose concentrations in liver and blood. The applied concentrations allowed to obtain the upper limits of quantification (both on chromatography and mass 
detector) (fig S8); the extended calibration curve showed saturation beyond the linear response of the GC-MS system. We then calculated the Response factor to adjust the new calibration to the original experimental run on a similar machine with identical settings. Finally, the measured glucose concentration were the linear range of the GC-MS system and thus glucose levels were absolutely quantified from the measured blood and liver samples. We measured glucose concentration in samples of blood taken from mice and naked mole-rats using a glucometer and found similar values to those calculated from the GC-MS experiment under basal conditions (fig S8).

The in-house-developed pSIRM-wizard enabled the quantification of ${ }^{13} \mathrm{C}$-label incorporation based on the exported data following the descriptions and equations previously described (2). The stable isotope incorporation was determined for a set of pre-defined metabolite-specific fragments. Mass isotopomer distributions (MID) for each fragment were calculated and exported by MAUI-Via. The measurement of non-labeled standards facilitated the correction for natural carbon abundance. Standard MIDs were automatically extracted from measurements matching the concentration range of the sample. The ${ }^{13} \mathrm{C}_{6}$-fructose incorporation in $\mathrm{CCM}$ intermediates has been calculated carbon-position resolved by the application of the targeted approach (2). Labeled quantities were determined for each time point by multiplying the ${ }^{13} \mathrm{C}$-incorporation of each measurement with the absolute metabolite pool. Statistical analysis was performed using a two-way ANOVA followed by Bonferroni post-hoc tests.

\section{Sequence alignment}

The amino acid sequences of guinea pig (H0VRF2), mouse (Q9WV38), rat (P43427) and human (P22732) GLUT5 were downloaded from UniProt (4), while naked mole-rat 
(NMR, XP_004863723.1) sequences were retrieved from the RefSeq database(5). Multiple alignment of the GLUT5 protein sequences was performed using the MUSCLE software package with default parameters (6).

\section{Ketohexokinase splice variant assay}

The Khk splicing assay was adapted from previously published protocols (7). RNA isolated from naked mole-rat and mouse liver tissue was reverse-transcribed then amplified using a forward primer (mouse; 5'-GAAGCAGATCCTGTGCGTG-3' and nmr; 5'-GAAGCGGATTCTGTGCGTG-3') that bound to shared $K h k \mathrm{~A} / \mathrm{C}$ exon 1 and a reverse primer (mouse; 5'-ATTGTGCTCCTCTATCCGC-3' and nmr; 5'GCGTATAGAACAGCACAAT-3') that bound to shared $K h k A / C$ exon 5 sequences. The 468-bp PCR products representing both $K h k-A$ and $K h k-C$ transcripts were then digested with HincII and run on a $1.5 \%$ agarose gel. The 468 bp represents the uncut Khk-Aspecific amplicon and the two lower bands at 294 bp and 174 bp represent HincII-cleaved Khk-C-specific amplicon.

\section{qPCR}

Total RNA was isolated from tissues using TRIzol reagent (Life Technologies), according to the manufacturer's instructions. After DNase digest, $0.65 \mu \mathrm{g}$ of total RNA was reverse transcribed with Supersript III reverse polymerase (Invitrogen) and analyzed using Universal Probe Library. Gene Expression Assays on the ABI 7900 system (Applied Biosystems). The following primers were used to quantify mouse Glut5 F: 5'AGAGCAACGATGGAGGAAAA-3' R: 5'-CCAGAGCAAGGACCAATGTC-3' and 
nmr Glut5 F: 5'-GTGCCCCAGCTCTTCATC-3' R:5'-GTTCCGAAAACCGAACAGC3', designed using the ProbeFinder Software with sequences retrieved for mouse (ENSMUST00000030826 ) and naked mole-rat (XM_004863666.1). To analyse KHK isoforms the following primer pairs were designed for mouse $K h k-A$ F: 5'TGGACTTACGATATGTGGTCCTT-3' R: 5'-GCCTCGTTGATGATGACTGTAG-3' and Khk-C F: 5'-GCGTGGATGTGTCTCAGGTG-3' R: TGTTGACGATGCAGCAAGA (reference sequence XM_006503749.1 and NM_008439.4, respectively.) and nmr Khk-A F: 5'-TCCGTGGACCTACGCTACTT-3' R: 5'-CTCGCTGATGATGACTGTGG-3' and nmr $\quad$ Khk-C F: 5'-CGTGGATGTGTCTCAGGTGT-3' and R: 5'AGATGTTGACGATGCAGCAG-3' (reference sequence XM_004839170.1 and XM_004839169.1, respectively). To calculate absolute numbers of transcripts, plasmids were made containing the cDNA amplicon from each primer pair for mouse and nmr. The standard curve method with known doses of plasmid was used to quantitate mRNA transcripts by extrapolating a value by comparing unknowns to the standard curve of known transcript amounts.

\section{Immunoblotting}

Tissues were lysed with RIPA buffer (50 mM Tris-HCL, pH 7.5, $150 \mathrm{mM} \mathrm{NaCl}, 1 \%$ Nonidet NP40, 0.5\% sodium deoxycholate, 1\% SDS) containing CHAPS (Sigma) and protein inhibitor cocktail (Roche) and protein concentration was determined using the Bradford reagent (BioRad). Thirty micrograms of proteins were separated by SDSPAGE, followed by Western blot analysis using rabbit Glut5 (SLC2A5) antibody (PA542100, Thermofischer), mouse GAPDH antibody (6C5, Calbiochem) and mouse anti $b$ actin (A1978, Sigma). Appropriate horseradish peroxidase-conjugated secondary 
antibodies were used for chemiluminescence (ECL, Millipore or Supra, Thermo scientific SuperSignal).

\section{Hippocampal Slice}

Experiments were performed with 2-4-month-old male C57Bl/6 mice and 1-4-year-old naked mole-rats. Maximum life span in naked mole-rats approaches 30 years; the animals used were considered to be adult, but not senescent. Animal protocols were approved by the University of Illinois at Chicago Institutional Animal Care and Use Committee.

Transverse hippocampal slices were prepared in the conventional manner. Briefly, mice and naked mole-rats were decapitated and the brains were rapidly removed into ice-cold artificial cerebrospinal fluid (ACSF) containing (in $\mathrm{mM}$ ): $\mathrm{NaCl}, 124 ; \mathrm{KCl}, 3 ; \mathrm{KH} 2 \mathrm{PO} 4$, 1.2; NaHCO3, 26; MgSO4, 2.5; CaCl2, 3.4; Na-ascorbate, 2; and D-glucose, 10, gassed with $95 \% \mathrm{O} 2$ and $5 \% \mathrm{CO} 2$. The tissue was then sliced at $400 \mu \mathrm{m}$ on a tissue chopper. Slices were placed in an interface chamber and constantly perfused $(1.0 \mathrm{ml} / \mathrm{min})$ with $\mathrm{ACSF}$ at 34-35C degrees for 1 hour. One mouse and one naked mole-rat were used on each day and slices from each were maintained in parallel in the same chamber for experiments. Stimulation electrodes were placed in the stratum radiatum of subfield CA1c to activate Schaffer-commissural fibers. Population recordings of synaptic field potentials (excitatory postsynaptic potentials or EPSPs) were made with micropipettes positioned in the stratum radiatum of CA1b. Evoked responses were digitized and analyzed online using custom software. Field EPSPs (fEPSPs) were evoked alternately in the mouse and naked mole-rats slices at $10 \mathrm{~s}$ intervals throughout experiments. Baseline stimulus intensity was set to evoke a half-maximal fEPSP in each slice. Baseline recordings were taken for at least 10 min before manipulations. Initial slope and peak 
amplitude were calculated for each fEPSP and normalized to the baseline average in each slice. For anoxic depolarization experiments, $\mathrm{O} 2$ to the recording chamber atmosphere and perfusion ACSF was replaced with $\mathrm{N}_{2}$. For the fructose experiments, a stable baseline was established then the perfusion solution was switched from the standard ACSF to an identical ACSF that replaced 10mM D-glucose with 10mM D-fructose-1,6bisphospate trisodium salt hydrate (Sigma Aldrich). After one hour the perfusion medium was switched back to standard ACSF and slices were recorded for 30 minutes to analyze recovery.

\section{Blood glucose and tissue glycogen measurement}

A drop of blood was obtained from a mouse via a tail nick and a prick to the foot in the naked mole-rat. Blood glucose was measured using an Accu-Check blood glucose meter

(Roche Diagnostics). Tissue glycogen was determined with a glycogen assay kit (Cayman Chemical) according to the manufacturer's instructions.

\section{Additional references}

1. V. Chu et al., Method for non-invasively recording electrocardiograms in conscious mice. BMC Physiol. 1, 6 (2001).

2. M. Pietzke, C. Zasada, S. Mudrich, S. Kempa, Decoding the dynamics of cellular metabolism and the action of 3-bromopyruvate and 2-deoxyglucose using pulsed stable isotope-resolved metabolomics. Cancer Metab. 2, 9 (2014). 
3. P. H. J. L. Kuich, N. Hoffmann, S. Kempa, Maui-VIA: a user-friendly software for visual identification, alignment, correction, and quantification of gas chromatography-mass spectrometry data. Bioinforma. Comput. Biol. 2, 84 (2015).

4. UniProt Consortium, UniProt: a hub for protein information. Nucleic Acids Res. 43, D204-212 (2015).

5. K. D. Pruitt et al., RefSeq: an update on mammalian reference sequences. Nucleic Acids Res. 42, D756-63 (2014).

6. R. C. Edgar, MUSCLE: multiple sequence alignment with high accuracy and high throughput. Nucleic Acids Res. 32, 1792-1797 (2004).

7. P. Mirtschink et al., HIF-driven SF3B1 induces KHK-C to enforce fructolysis and heart disease. Nature. 522, 444-9 (2015). 


\section{Supplementary Figures}

A

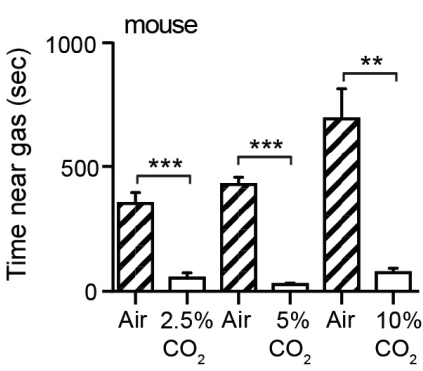

C

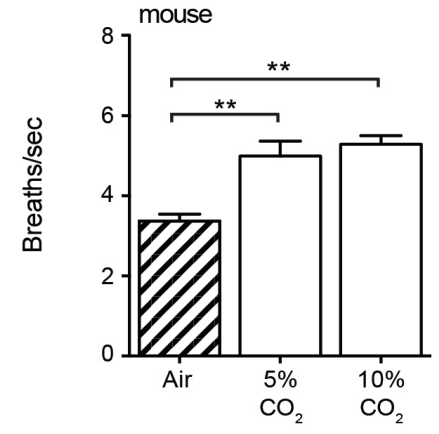

E

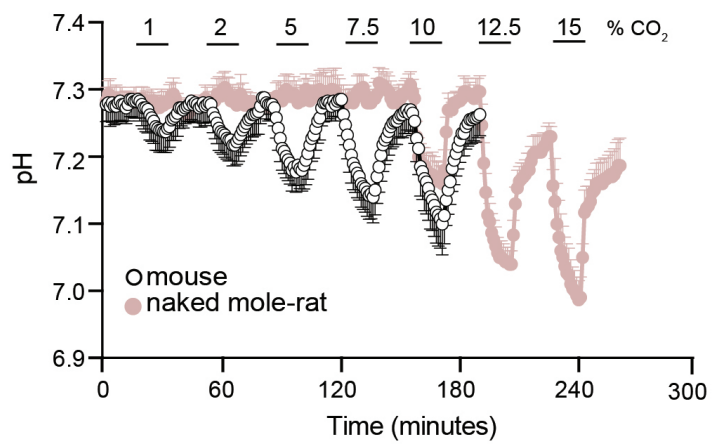

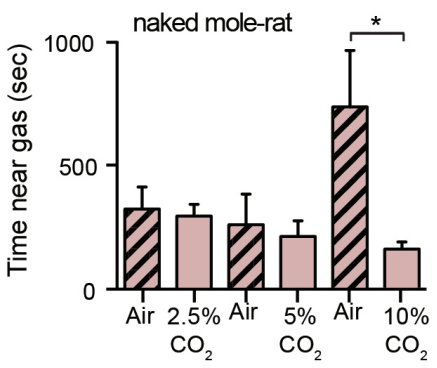

D

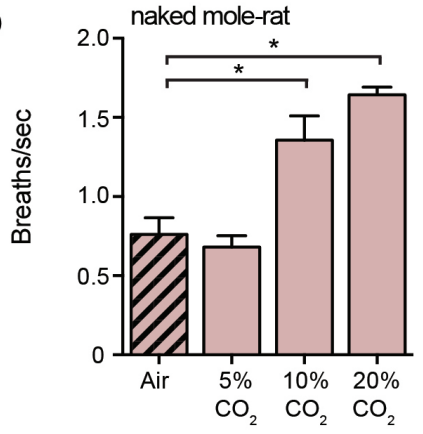

$\mathbf{F}$

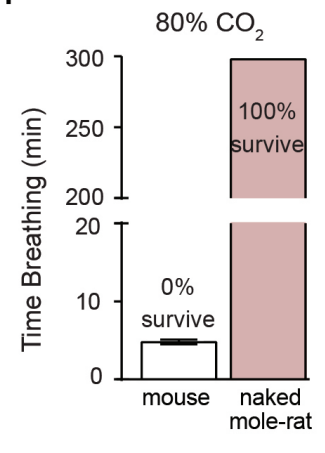

Figure S1 Reduced physiological response to hypercapnia in naked mole-rats (A) Mice show robust avoidance of $\mathrm{CO}_{2}$ presented at concentrations shown. (B) Naked molerats do not avoid $\mathrm{CO}_{2}$ gas until concentrations reach $10 \%$. (C) $\mathrm{CO}_{2}$ presented at 5 and $10 \%$ induces robust hyperventilation in mice. (D) Only $\mathrm{CO}_{2}$ concentrations of $10 \%$ and above induce hyperventilation in naked mole-rats. (E) A pH electrode was placed in the abdomen to measure systemic extracellular $\mathrm{pH}$. Inhaled $\mathrm{CO}_{2}$ concentrations of $1 \%$ and above induce prominent tissue acidosis in mice but in naked mole-rats only $\mathrm{CO} 2$ concentrations above $7.5 \%$ induced acidosis. (F) Time breathing in $80 \% \mathrm{CO}_{2} / 20 \% \mathrm{O}_{2}$ for naked mole-rats and mice (cut-off time 300 mins). Mice did not survive any procedure whereas naked mole-rats always survived. Data shown as mean \pm S.E.M. ${ }^{*} \mathrm{p}<0.05$, $* * \mathrm{p}<0.01, * * * \mathrm{p}<0.001$ Student's t-test, $\mathrm{n}=4-6$. 
A

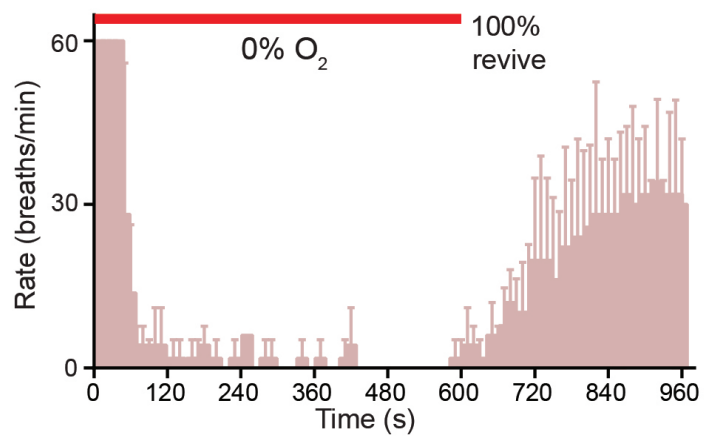

B

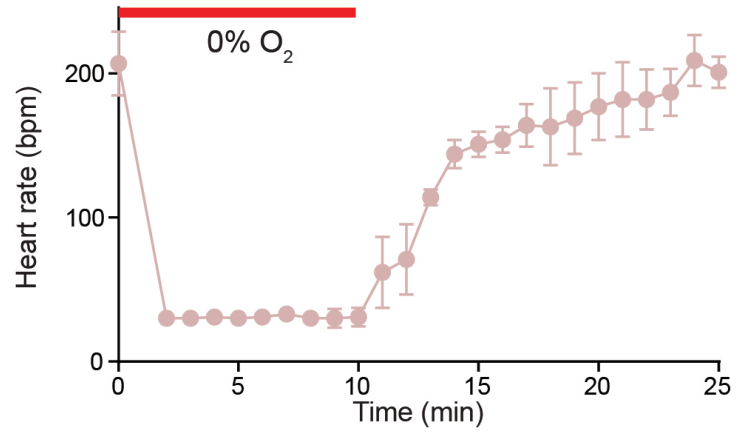

Figure S2 Anoxia resistance in naked mole-rats after a 10 minute exposure

(A) Respiration rate and (B) heart rate during $10 \mathrm{~min}$ of $0 \% \mathrm{O}_{2}$ showed a pattern of drastic reduction followed by recovery in the naked mole-rat. Note heart rate recovered fater after exposure to room air than in the 18 min group (Fig 1D,E).

A

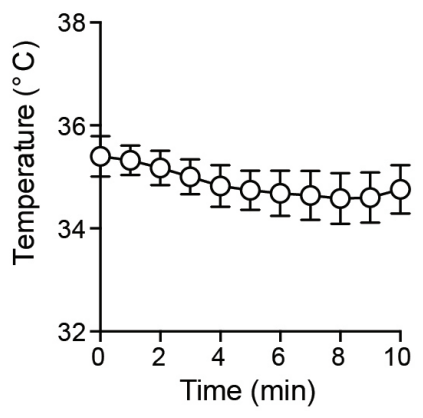

C

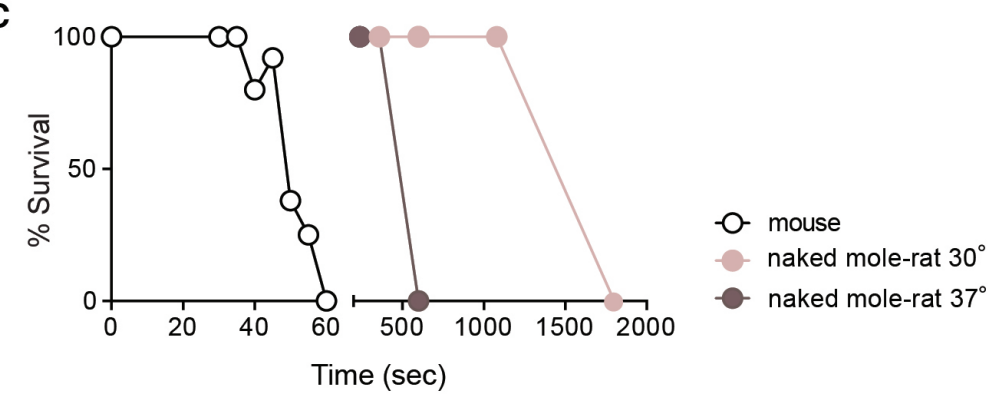

Figure S3 Temperature dependence of anoxia resistance in naked mole-rats

(A) Body temperature of mice during 10 mins of $5 \% \mathrm{O}_{2}$ exposure. (B) Body temperature of naked mole-rats kept at $30^{\circ}$ during 10 mins of anoxia exposure. (C) Survival curves of mice and naked mole-rats maintained at $30^{\circ}$ or $37^{\circ}$ in $0 \% \mathrm{O}_{2}$. Naked mole-rats kept at $37^{\circ}$ all survived 6 mins of anoxia exposure $(\mathrm{n}=3)$ but not 10 mins anoxia exposure compared to naked mole-rats at $30^{\circ}$ which survived up to 18 minutes anoxia $(n=3)$. 
A

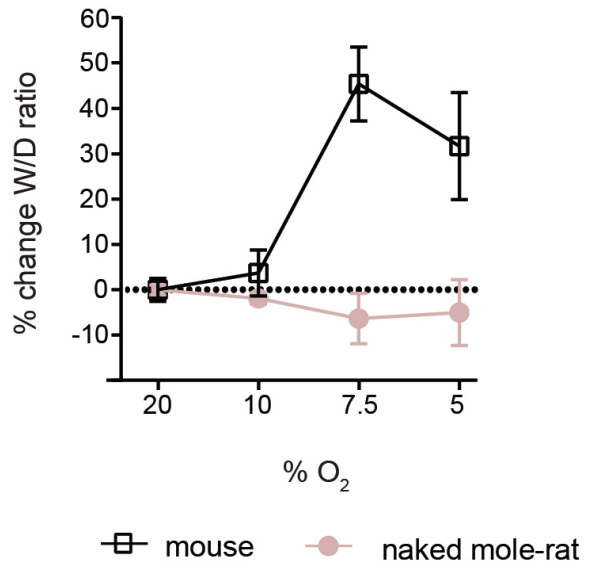

B

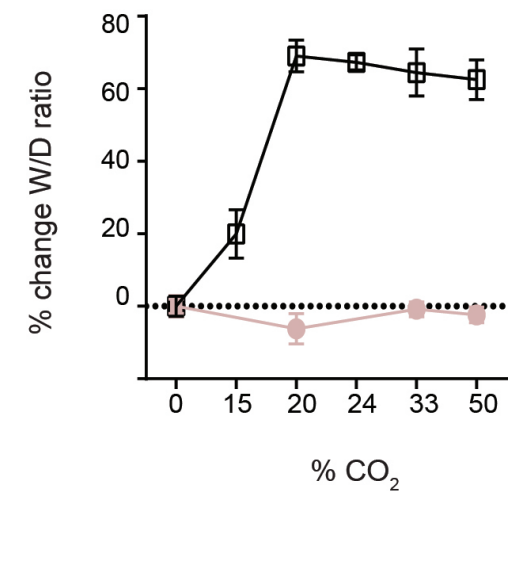

Figure S4 Pulmonary edema in mice and naked mole-rats during hypoxia and hypercapnia. The percentage change in the wet to dry weight ratio (W/D) of lung tissue was measured to assess lung edema after exposure to increasing hypoxia or hypercapnia. (A) Exposure to less than $10 \% \mathrm{O}_{2}$ produced severe pulmonary edema in mice but produced essentially no edema in naked mole-rats $(\mathrm{n}=3)$. (B) Concentrations of $\mathrm{CO}_{2}$ greater than $10 \%$ also caused severe pulmonary edema in mice which was maximal above $20 \% \mathrm{CO}_{2}$. No signs of edema were observed in naked-mole-rat lung at $\mathrm{CO}_{2}$ up to $50 \%(n=3)$. 
A BLOOD
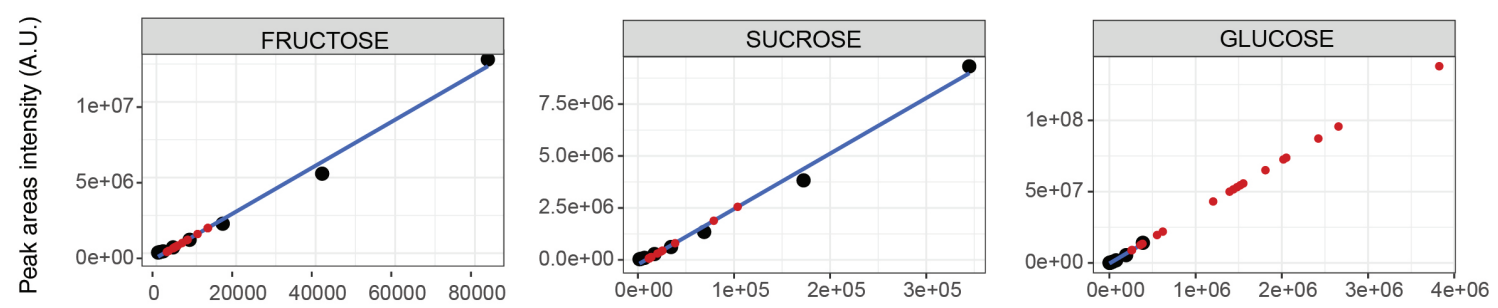

B LIVER and BRAIN
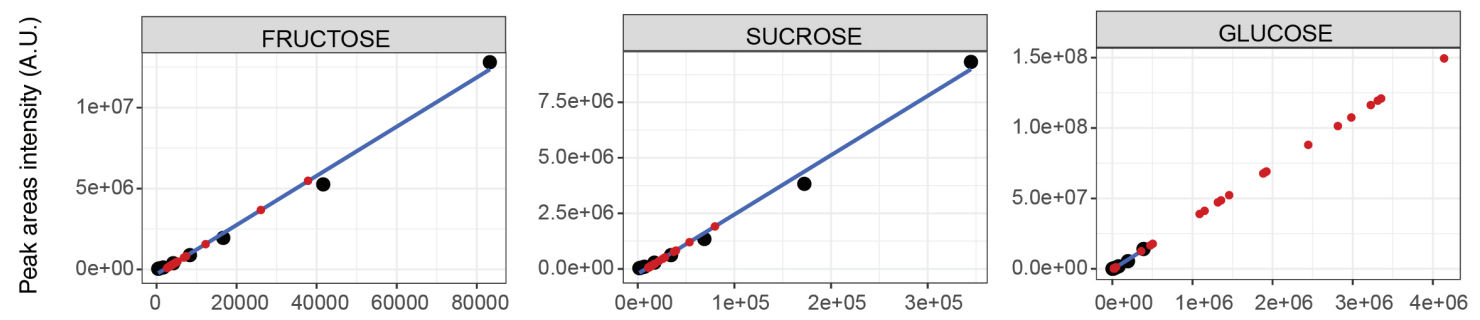

C

KIDNEY and MUSCLE
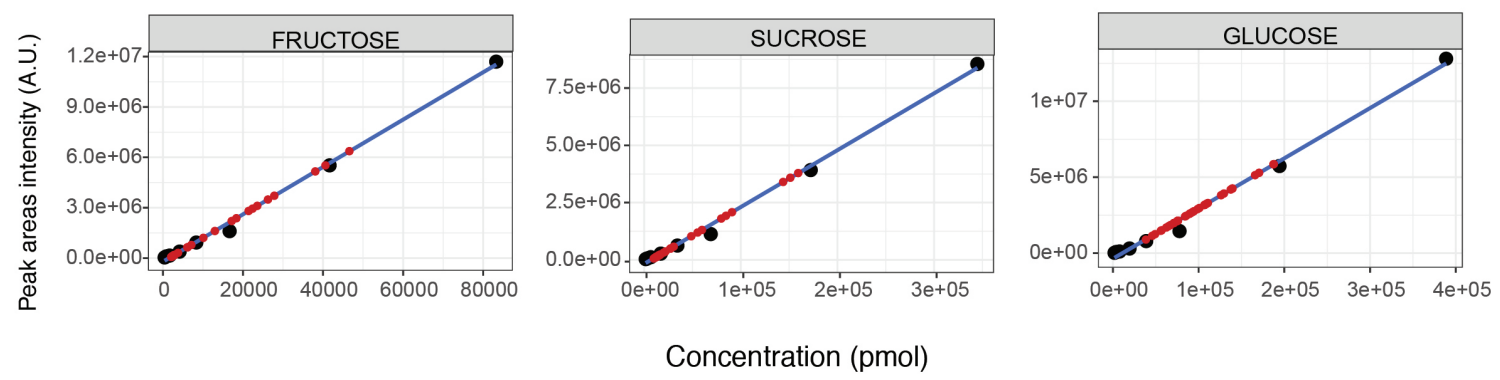

Figure S5 Calibration curves for absolute quantification of metabolites

Quantification standards (black circles) used for calibration curves to measure samples (red circles) in absolute concentrations for fructose, sucrose and glucose in (A) blood (B) liver and brain and (C) kidney and muscle. Note that glucose in blood and liver samples fell outside of the quantifiable linear part of the calibration curve in the first experiment. However, the samples were measured once more with a higher concentrations of glucose standards, the absolute values for glucose (plotted in fig S8) were then recalculated from these extended calibration curves. 
A
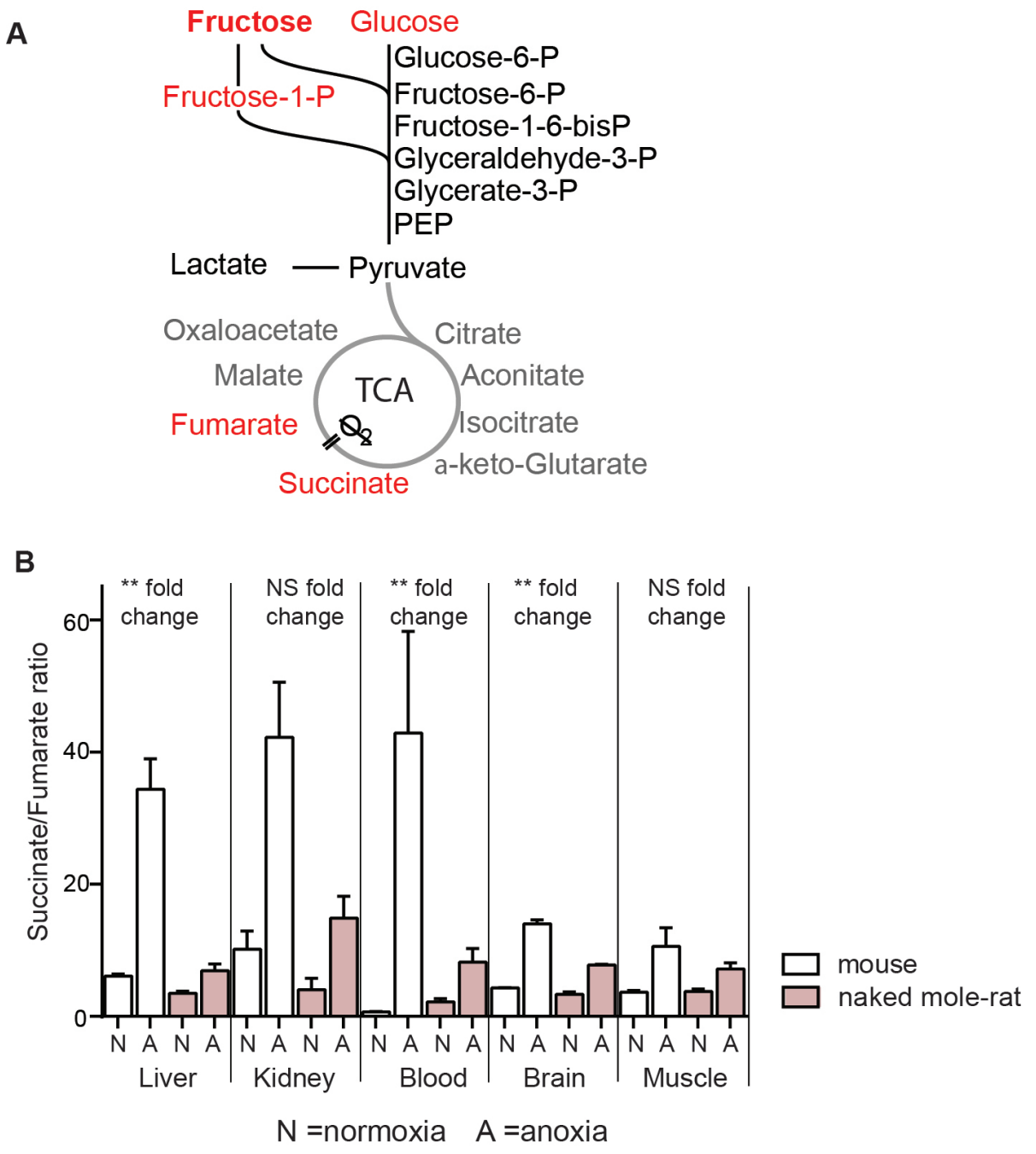

Figure S6 Suppression of mitochondrial metabolism in anoxia exposed naked molerats. (A) Schematic of glycolysis and TCA cycle where in the absence of $\mathrm{O}_{2}$ succinate cannot be converted to fumarate. (B) Large increases in the succinate to fumarate ratio were observed in mouse liver, kidney blood and brain and these fold changes were significantly larger than those observed in naked mole-rat tissue after 10 min exposure to $0 \% \mathrm{O}_{2}$. Data shown as mean \pm S.E.M. ${ }^{*} \mathrm{p}<0.01$ Student's T-test, $\mathrm{n}=3$. 
A

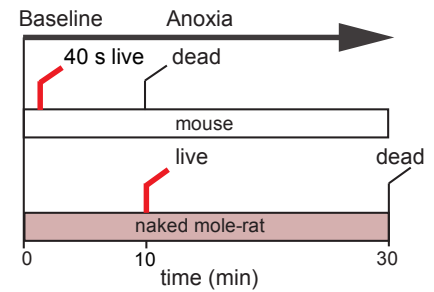

B
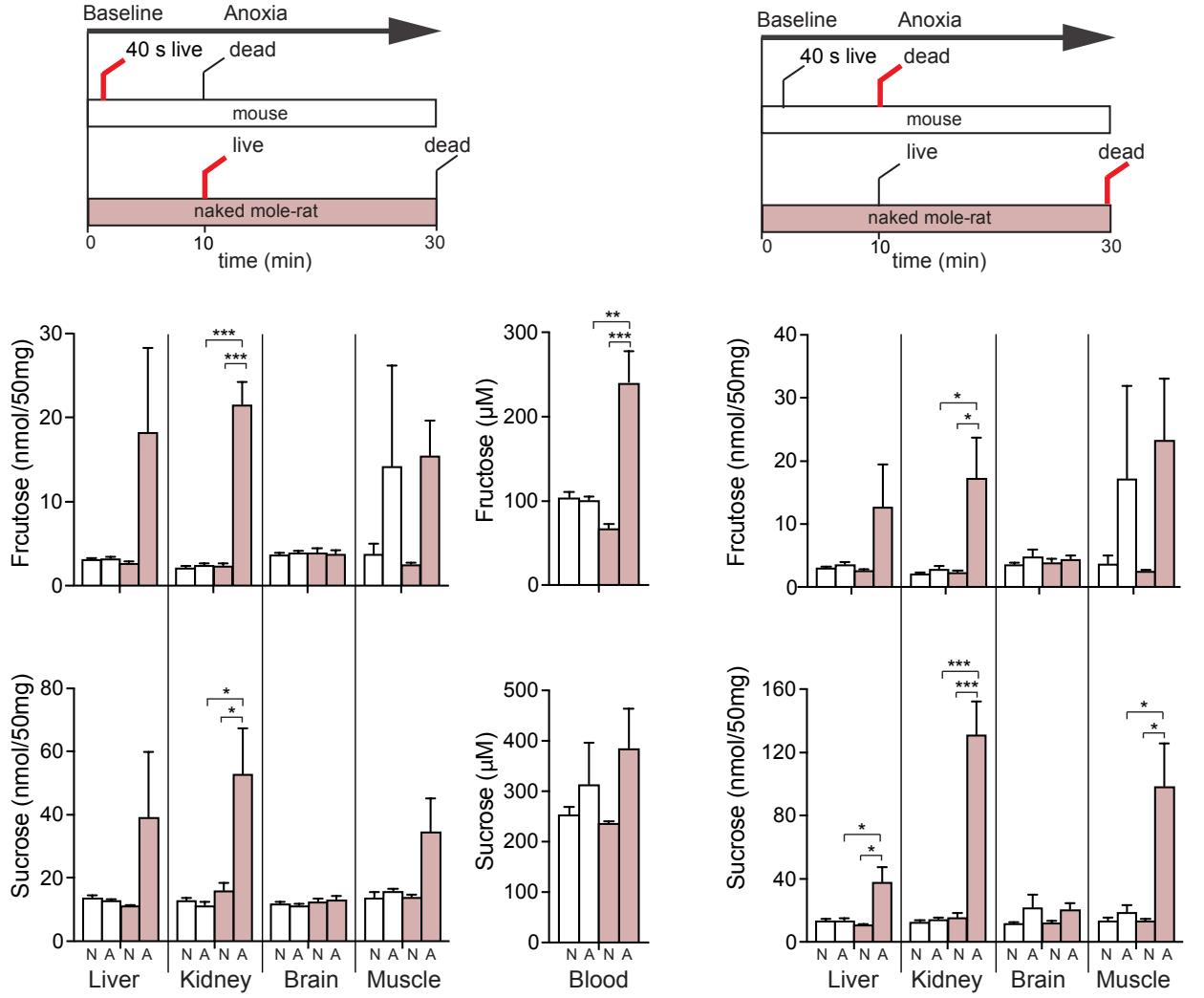
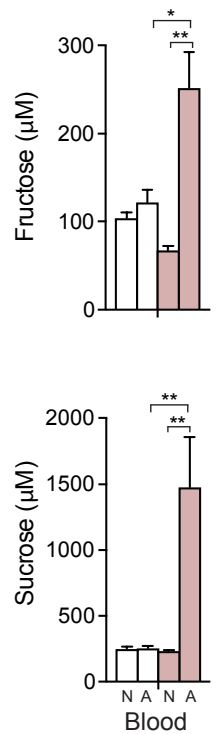

$\mathrm{N}=$ normoxia $\quad \mathrm{A}=$ =anoxia

mouse

$\square$ naked mole-rat

Figure S7 Increases in fructose and sucrose in tissues of naked mole-rats during exposure to anoxia. Absolute concentrations of fructose and sucrose in the liver, kidney, blood, brain and muscle of mice and naked mole-rats in normoxia compared to (A) $40 \mathrm{~s}$ anoxia for mouse and 10 mins anoxia for naked mole-rats (live:live comparison) and (B) 10 mins anoxia for mouse and 30 mins anoxia for naked mole-rat (dead:dead comparison). Data shown as mean \pm S.E.M. ${ }^{*} \mathrm{p}<0.05 ;{ }^{* *} \mathrm{p}<0.01,{ }^{* * *} \mathrm{p}<0.001$ using a 2-WAY ANOVA with Tukey's post-hoc test, $\mathrm{n}=3$. 

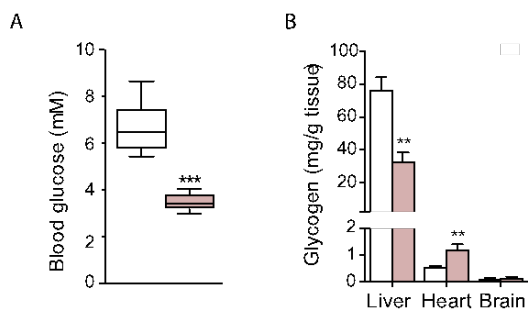

$c$
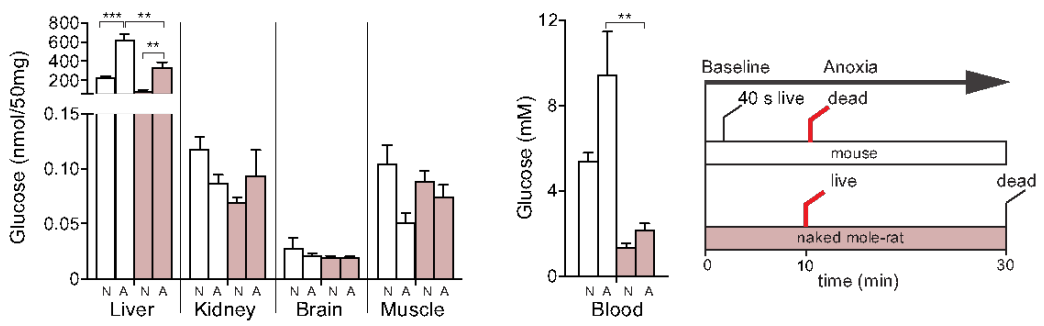

D
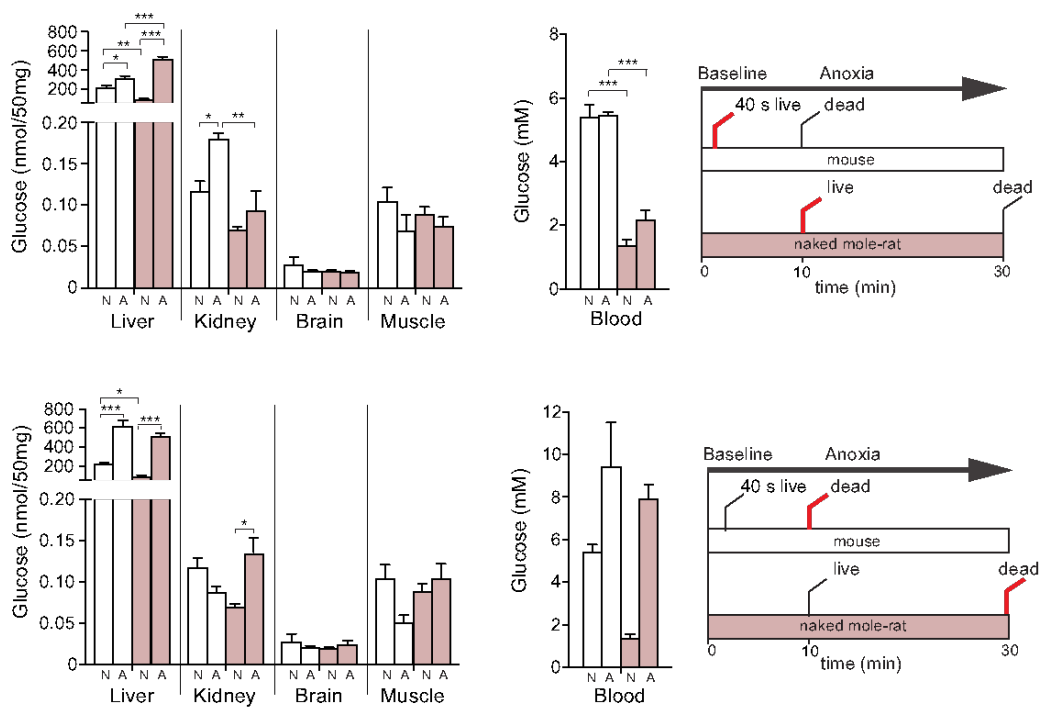

Figure S8 Glucose concentration in mouse and naked mole-rat during anoxia exposure. (A) Blood glucose concentrations in mouse versus naked mole-rat. Data shown as mean \pm S.E.M. ***p $<0.001$ Student's $\mathrm{t}$-test, $\mathrm{n}=10$. (B) Glycogen concentrations in liver, heart and brain in mice and naked mole-rats. Data shown as mean \pm S.E.M. ${ }^{* *} \mathrm{p}<0.01$ Student's $\mathrm{t}-$ test, $\mathrm{n}=3-6$. Changes in glucose in the kidney, brain and muscle, liver and blood in mice and naked mole-rats in normoxia compared to (C) 10 mins anoxia for mice and naked mole-rats (D) $40 \mathrm{~s}$ anoxia for mouse and 10 mins anoxia for naked mole-rats (live:live comparison) and (E) 10 mins anoxia for mouse and 30 mins anoxia for naked mole-rat (dead:dead comparison). Data shown as mean \pm S.E.M. *p $<$ $0.05 ; *$ p $<0.01, * * * p<0.001$ using a 2-WAY ANOVA with Tukey's post-hoc test, $\mathrm{n}=3$. 
A
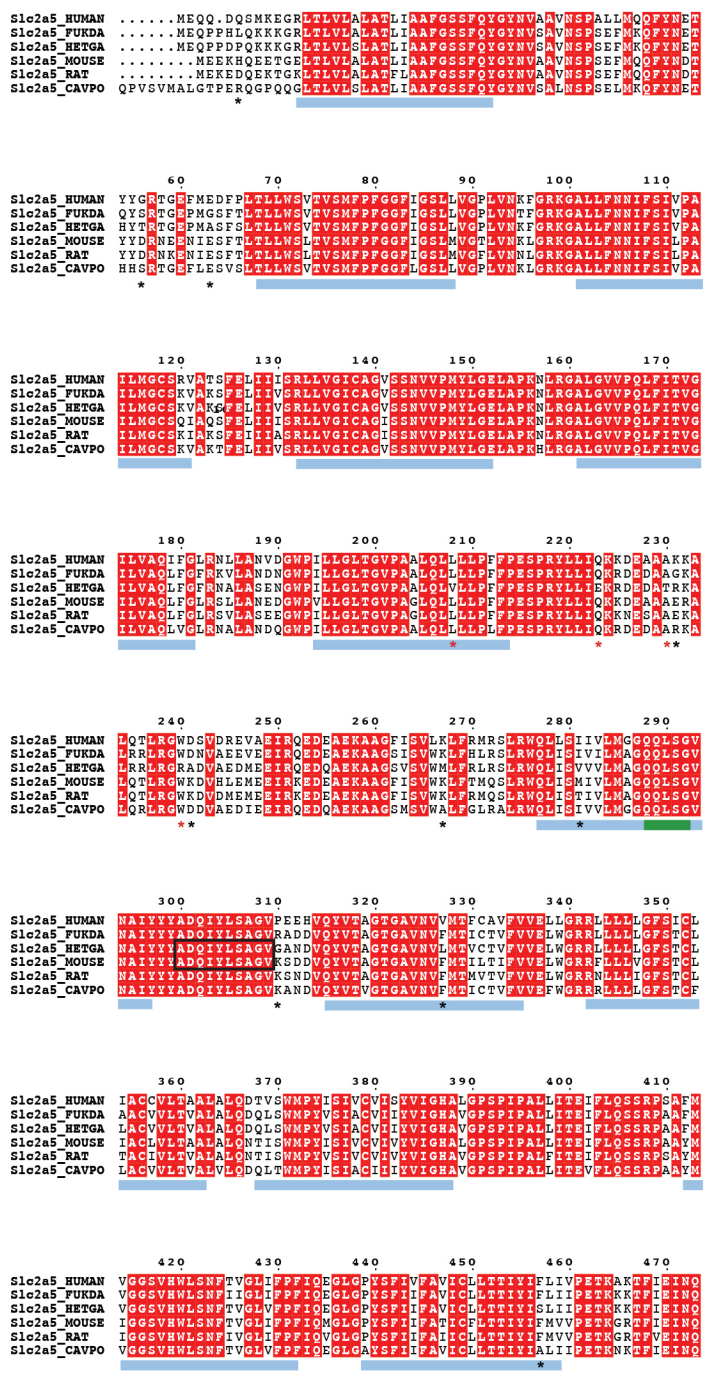

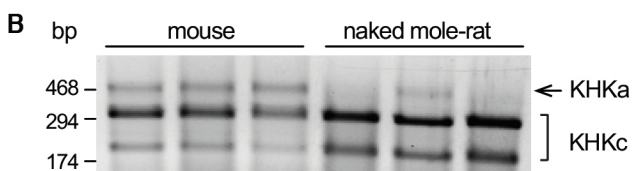

C

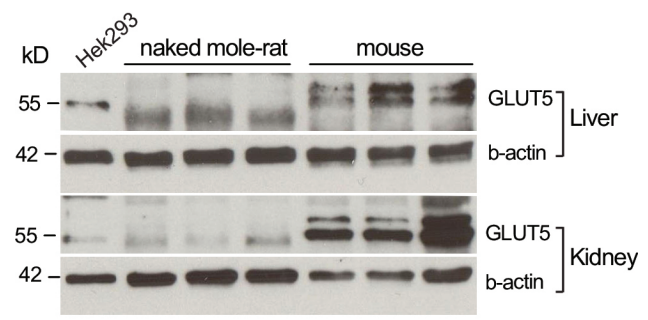

Figure S9 Multiple sequence alignment of the GLUT5 protein. (A) Alignments are shown from the guinea pig (CAVPO), mouse (MOUSE), rat (RAT), Damaraland mole rat (FUKDA), naked mole-rat (HETGA), and human proteins (HUMAN). Bars in blue indicate transmembrane domains, green bar indicates the suggested fructose binding site. Residues marked with black asterisks appear to be variants that are unique to naked molerats. Red asterisks indicate amino acid variants that are naked mole-rat specific but are in regions where the residue is conserved amongst the other species. Black box outlines the conserved epitope of the GLUT5 antibody (B) KHK-A and KHK-C isoform expression in the liver (PCR followed by HincII digestion). Upper band is the uncut KHK-A isoform, lower bands are the cut KHK-C isoform. (C) GLUT5 protein in Hek293 cells, naked mole-rat and mouse liver and kidney. Naked mole-rat GLUT5 protein runs slightly lower than $55 \mathrm{kD}$ whereas mouse GLUT5 appears as a double band. Protein content was controlled for by blotting for b-actin $(n=3)$. 


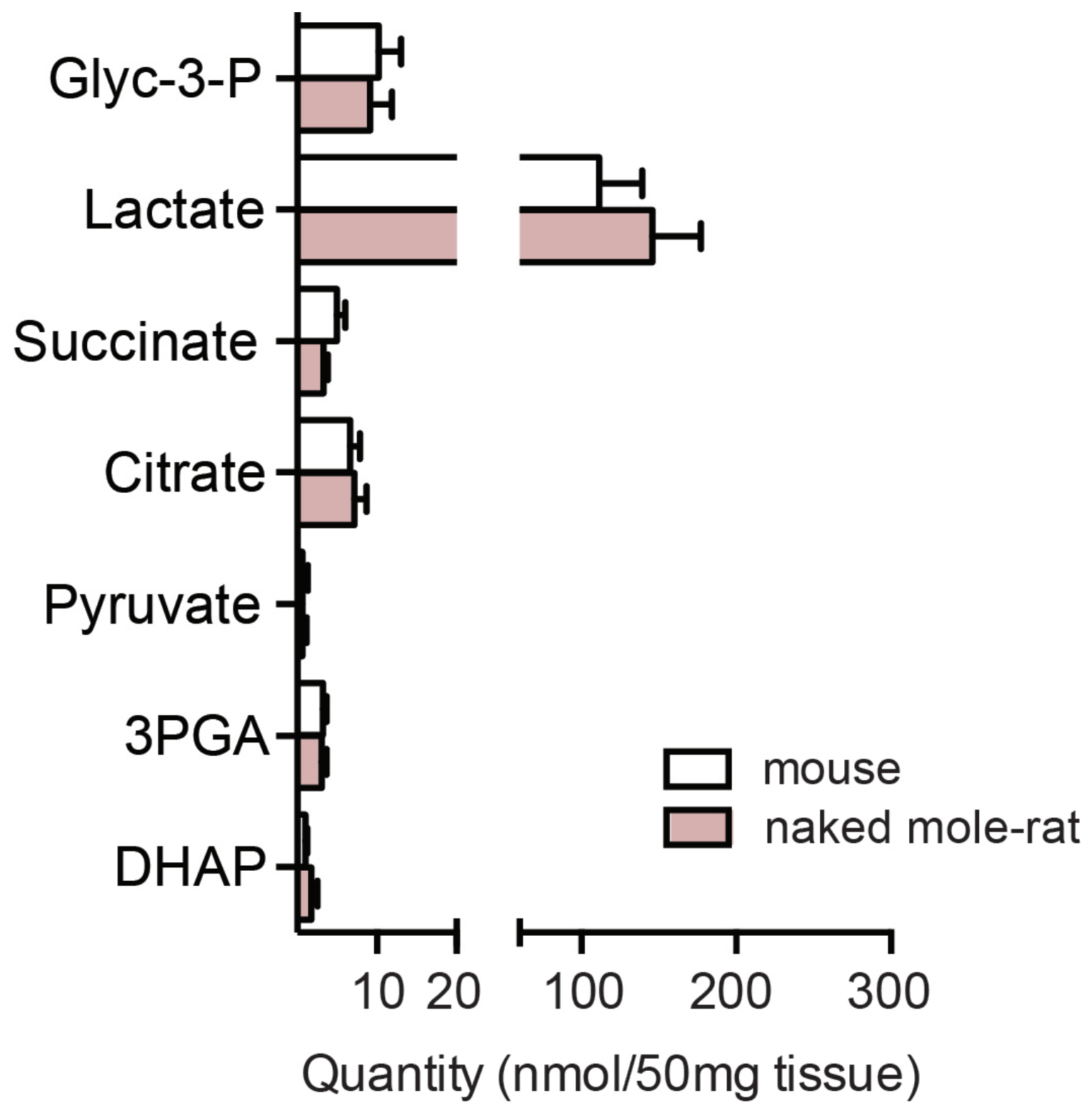

Figure S10 Absolute metabolite pools in mouse and naked mole-rat brain slices determined by GC-MS analysis. DHAP; Dihydroxyacetone phosphate, Glyceraldehyde-3-phosphate, 3-PGA; 3-Phosphoglyceric acid. $(\mathrm{n}=12$; error bars are S.E.M.) 


\section{Table S1}

Comparison of heart parameters in naked mole-rat vs. mouse ( $\mathrm{Mm} \mathrm{C57BI/6)}$ at $30^{\circ}$ and $37^{\circ} \mathrm{C}$

回

\begin{tabular}{|c|c|c|}
\hline 田 & ARTERTE & 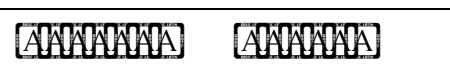 \\
\hline 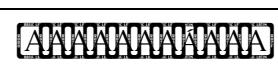 & 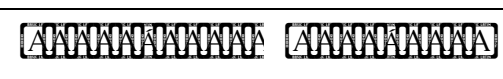 & 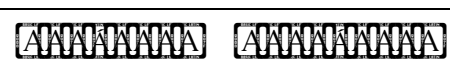 \\
\hline
\end{tabular}

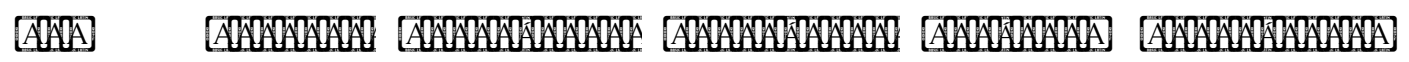

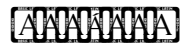

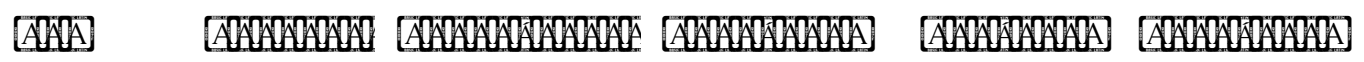

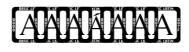

田

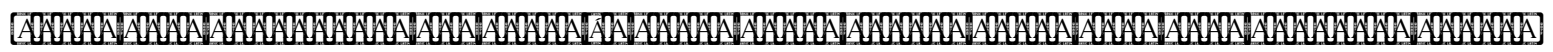

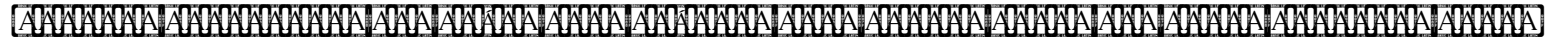

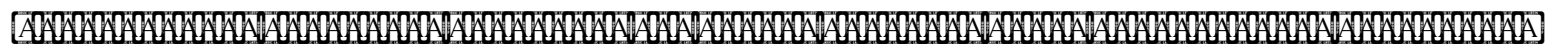

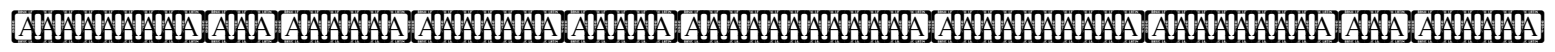

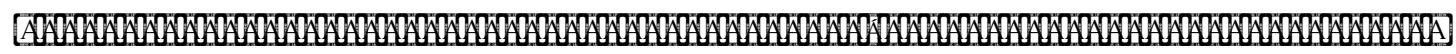

曰 田 
Additional Data Table S1

Excel Datasheet containing mean intensity values for metabolites measured. 